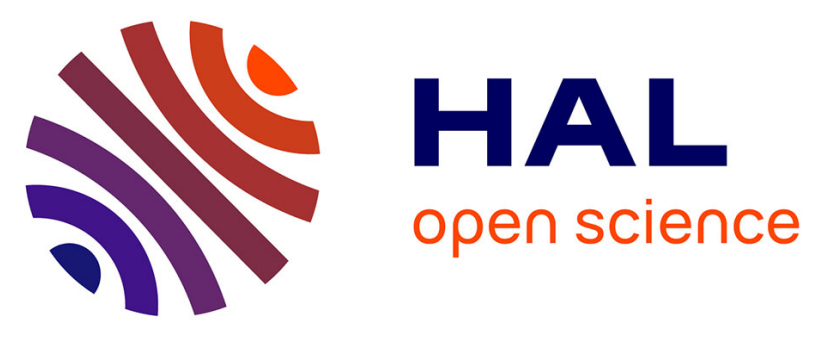

\title{
Understanding Active Sites in Pyrolyzed Fe-N-C Catalysts for Fuel Cell Cathodes by Bridging Density Functional Theory Calculations and 57 Fe Mössbauer Spectroscopy
}

Tzonka Mineva, Ivana Matanovic, Plamen Atanassov, Moulay Tahar Sougrati, Lorenzo Stievano, Martin Clémancey, Amélie Kochem, Jean-Marc Latour, Frédéric Jaouen

\section{- To cite this version:}

Tzonka Mineva, Ivana Matanovic, Plamen Atanassov, Moulay Tahar Sougrati, Lorenzo Stievano, et al.. Understanding Active Sites in Pyrolyzed Fe-N-C Catalysts for Fuel Cell Cathodes by Bridging Density Functional Theory Calculations and 57 Fe Mössbauer Spectroscopy. ACS Catalysis, 2019, 9 (10), pp.9359-9371. 10.1021/acscatal.9b02586 . hal-02292379

HAL Id: hal-02292379

https://hal.science/hal-02292379

Submitted on 16 Nov 2020

HAL is a multi-disciplinary open access archive for the deposit and dissemination of scientific research documents, whether they are published or not. The documents may come from teaching and research institutions in France or abroad, or from public or private research centers.
L'archive ouverte pluridisciplinaire HAL, est destinée au dépôt et à la diffusion de documents scientifiques de niveau recherche, publiés ou non, émanant des établissements d'enseignement et de recherche français ou étrangers, des laboratoires publics ou privés. 


\section{Understanding active sites in pyrolyzed Fe-N-C catalysts for fuel cell cathodes by bridging density functional theory calculations and ${ }^{57} \mathrm{Fe}$ Mössbauer Spectroscopy}

Tzonka Mineva $^{\mathrm{a}^{*}}$, Ivana Matanovic ${ }^{\mathrm{b}, \mathrm{c}}$, Plamen Atanassov ${ }^{\mathrm{b}, \mathrm{d}}$, Moulay-Tahar Sougrati ${ }^{\mathrm{a}}$, Lorenzo Stievano $^{\mathrm{a}}$, Martin Clémancey ${ }^{\mathrm{e}}$, Amélie Kochem, ${ }^{\mathrm{e}}$ Jean-Marc Latour ${ }^{\mathrm{e}}$, Frédéric Jaouen ${ }^{\mathrm{a} *}$

a Institut Charles Gerhardt Montpellier, UMR 5253, CNRS, Université Montpellier, ENSCM, 34090 Montpellier, France

${ }^{\mathrm{b}}$ The Department of Chemical and Biological Engineering, Center for Micro-Engineered Materials (CMEM), University of New Mexico, Albuquerque, NM 87131, USA.

${ }^{\mathrm{c}}$ Theoretical Division, Los Alamos National Laboratory, Los Alamos, NM 87545, USA

${ }^{\mathrm{d}}$ Chemical \& Biomolecular Engineering and National Fuel Cell Research Center, University of California, Irvine, CA 92697-2580, USA

${ }^{\text {e }}$ Université Grenoble Alpes CNRS, CEA, DRF/IRIG/LCBM/pmb, 17 rue des Martyrs, 38000 Grenoble, France

emails : tzonka.mineva@enscm.fr, igonzales@unm.edu, moulaytahar.sougrati@umontpellier.fr,$\underline{\text { lorenzo.stievano@umontpellier.fr }}$, plamen.atanassov@uci.edu, martin.clemancey@cea.fr, amelie.kochem@cea.fr, jean$\underline{\text { marc.latour@cea.fr, frederic.jaouen@umontpellier.fr }}$

*Corresponding authors: tzonka.mineva@enscm.fr; frederic.jaouen@umontpellier.fr 


\begin{abstract}
Pyrolyzed Fe-N-C materials are promising platinum-group-metal free catalysts for protonexchange membrane fuel cell cathodes. However, the detailed structure, oxidation and spin states of their active sites is still undetermined. ${ }^{57} \mathrm{Fe}$ Mössbauer spectroscopy has identified $\mathrm{FeN}_{\mathrm{x}}$ moieties as the most active sites, with their fingerprint being a doublet in lowtemperature Mössbauer spectra. However, the interpretation of the doublets for such materials has lacked theoretical basis. Here, we applied density functional theory to calculate the quadrupole splitting energy of doublets $\left(\Delta E_{\mathrm{QS}}\right)$ for a range of $\mathrm{FeN}_{\mathrm{x}}$ structures in different oxidation and spin states. The calculated and experimental values are then compared for a reference $\mathrm{Fe}-\mathrm{N}-\mathrm{C}$ catalyst, while further information on the Fe oxidation and spin states was obtained from electron paramagnetic resonance, superconducting quantum interference device and ${ }^{57} \mathrm{Fe}$ Mössbauer spectroscopy under external magnetic field. The combined theoretical and experimental results identify the main presence of $\mathrm{FeN}_{\mathrm{x}}$ moieties in $\mathrm{Fe}$ (II) low-spin and $\mathrm{Fe}(\mathrm{III})$ high-spin states while a minor fraction of sites could exist in $\mathrm{Fe}(\mathrm{II}) \mathrm{S}=1$ state. From the analysis of the ${ }^{57} \mathrm{Fe}$ Mössbauer spectrum under external magnetic field and the comparison of calculated and measured $\Delta E_{\mathrm{QS}}$ values, we assign the experimental doublet D1 with mean $\Delta E_{\mathrm{QS}}$ value around $0.9 \mathrm{~mm} \cdot \mathrm{s}^{-1}$ to $\mathrm{Fe}(\mathrm{III}) \mathrm{N}_{4} \mathrm{C}_{12}$ moieties in high spin and the experimental doublet D2 with mean $\Delta E_{\mathrm{QS}}$ value around $2.3 \mathrm{~mm} \cdot \mathrm{s}^{-1}$ to $\mathrm{Fe}(\mathrm{II}) \mathrm{N}_{4} \mathrm{C}_{10}$ moieties in low and medium spin. These conclusions indicate that D1 corresponds to surface-exposed sites while D2 may correspond either to bulk sites that are inaccessible to $\mathrm{O}_{2}$ or to surface sites that bind $\mathrm{O}_{2}$ weaker than $\mathrm{D} 1$.
\end{abstract}

\title{
Keywords
}

Density functional theory, Mössbauer spectroscopy, quadrupole energy splitting, $\mathrm{FeN}_{4}, \mathrm{Fe}-\mathrm{N}$ $\mathrm{C}$, oxygen reduction, fuel cell, cathode 


\section{Introduction}

${ }^{57} \mathrm{Fe}$ Mössbauer spectroscopy, based on the recoil-free emission and resonant absorption of $\gamma$ rays, has been extensively used to fingerprint the local electronic structure, magnetic properties and coordination of iron nuclei in different classes of important materials ranging from (extra) terrestrial minerals and biomolecules containing $\mathrm{Fe}$ in their active site, to industrial catalysts such as those used for Fischer-Tropsch or ammonia synthesis. ${ }^{1-4}$ More recently, it has gained growing importance in the characterization of novel platinum-free catalysts for application in fuel cell cathodes for the identification of Fe-based species present in pyrolyzed Fe-N-C catalysts. ${ }^{5-17}$. Due to their synthetic approach involving a high temperature treatment, such catalysts often comprise a plurality of Fe-based structures, including not only different coordination geometries of atomically-dispersed $\mathrm{Fe}$ ions covalently bound to the nitrogen-doped carbon matrix $\left(\mathrm{FeN}_{\mathrm{x}} \mathrm{C}_{\mathrm{y}}\right.$ moieties), but also metallic iron and iron carbides with long-range order. Compared to other spectroscopic methods and to X-ray absorption in particular, ${ }^{57} \mathrm{Fe}$ Mössbauer spectroscopy allows an easier separation of the signals arising from the different Fe environments. Namely, for spectra recorded in the absence of a strong external magnetic field, metallic iron and iron carbides result in sextets (except for the paramagnetic $\gamma$-Fe structure, leading to a singlet) while the fingerprint of $\mathrm{FeN}_{\mathrm{x}} \mathrm{C}_{\mathrm{y}}$ moieties is a quadrupole doublet. Its two Mössbauer parameters are the isomer shift $(\delta)$ and the quadrupole splitting energy $\left(\Delta E_{\mathrm{QS}}\right) .{ }^{18-20}$ These two parameters originate from hyperfine interactions between the iron nucleus and the surrounding electronic environment, and vary therefore with the iron oxidation state, spin state, and its chemical surrounding. ${ }^{21}$

Due to the clear different shape and Mössbauer parameters of the spectral signals arising from different Fe structures, the fitting of the experimental spectrum of any $\mathrm{Fe}-\mathrm{N}-\mathrm{C}$ catalyst with a number of spectral components (singlets, doublets and sextets) is usually relatively straightforward and gives direct quantitative information on the fraction of Fe present in different forms. However, while the spectral parameters found for the singlet and sextet components identified in the spectra of pyrolyzed Fe-N-C catalysts perfectly match with those known for the well-established Fe crystalline structures (metallic Fe, Fe carbides, Fe nitrides), leading to unambiguous assignments, the interpretation of the spectral parameters fitted for the doublets in such materials have so far lacked strong bases, both from the experimental and theoretical sides. The lack of appropriate reference materials (materials with a single type of $\mathrm{FeN}_{\mathrm{x}} \mathrm{C}_{\mathrm{y}}$ moieties in a carbon matrix, with the local structure around $\mathrm{Fe}$ atoms being 
independently determined) prohibits making unambiguous assignments on the experimental side. The Mössbauer spectral parameters of some Fe macrocycles with known structure (iron phthalocyanines and porphyrins, pure or adsorbed on carbon surfaces) have hitherto been used to make assignments of the various doublets typically observed in pyrolyzed Fe-N-C materials to ferrous or ferric iron in different spin states. ${ }^{6}$ The assumption that such macrocycles may serve as proper models for all or some of the $\mathrm{FeN}_{\mathrm{x}} \mathrm{C}_{\mathrm{y}}$ moieties present in pyrolyzed Fe-N-C materials is however too speculative, for several reasons. First, the size of these reference molecules is much smaller than the average in-plane size of graphitic crystallites in pyrolyzed Fe-N-C materials (typically $2 \mathrm{~nm}$ and above). Second, such macrocycles with $\mathrm{FeN}_{4}$ core moiety involve pyrrolic nitrogen atoms engaged in fivemembered rings, while perfectly graphitized materials comprise only six-membered hexagonal rings. Experimental proof for the existence of atomically-dispersed Fe atoms in a nitrogen-doped carbon matrix has recently been confirmed by high-resolution electron microscopy, both in amorphous carbons (comprising a significant fraction of non-hexagonal rings) and in perfectly ordered graphene sheets (comprising only hexagonal rings). ${ }^{22-23}$ Moreover, even for an Fe-N-C model catalyst with amorphous carbon matrix and comprising only atomically-dispersed Fe atoms and whose XANES and EXAFS spectra could be fitted with a single porphyrinic $\left(\mathrm{FeN}_{4} \mathrm{C}_{12}\right)$ structure, the fitting of its Mössbauer spectrum still required two distinct doublets, at least. ${ }^{10}$ Third, the $\mathrm{FeN}_{\mathrm{x}} \mathrm{C}_{\mathrm{y}}$ moieties in pyrolyzed materials are integrated in a conductive carbon matrix, which may completely change the electron density at the $\mathrm{Fe}$ nucleus relative to an adsorbed $\mathrm{Fe}$ macrocycle, even for a similar local coordination. As discussed below, the electron-density at the Fe nucleus is a key in determining the spectral Mössbauer parameters. Hitherto, all pyrolyzed $\mathrm{Fe}-\mathrm{N}-\mathrm{C}$ catalysts comprising $\mathrm{FeN}_{\mathrm{x}} \mathrm{C}_{\mathrm{y}}$ moieties have shown at least two distinct doublets in their Mössbauer spectra, often labeled D1 and D2. Their reported values of $\Delta E_{\mathrm{QS}}$ at room temperature are in the range of $0.90-1.25$ and $2.0-2.8 \mathrm{~mm} \cdot \mathrm{s}^{-1}$, respectively, while their isomer shifts are comparable and non-discriminating $\left(0.30-0.45 \mathrm{~mm} \cdot \mathrm{s}^{-1}\right){ }^{24}$ The doublet D1 has, most frequently, been empirically assigned to an $\mathrm{Fe}(\mathrm{II})$ zero spin $(\mathrm{S}=0)$ moiety, ${ }^{6}{ }^{9}$ but also sometime to $\mathrm{Fe}(\mathrm{III})$ in high spin $(\mathrm{S}=5 / 2) .{ }^{5,8}$ The doublet $\mathrm{D} 2$ has been empirically assigned either to an distorted Fe(II) moiety in low spin, Fe(II) medium spin ( $\mathrm{S}=1)$, Fe(III) low spin $(\mathrm{S}=1 / 2)$ or $\mathrm{Fe}(\mathrm{III})$ medium spin $(\mathrm{S}=3 / 2)$ moiety. ${ }^{6,9}$ These empirical attributions are however not yet supported by any theory or independent experimental method. This uncertainty casts a doubt on past interpretation of the ${ }^{57} \mathrm{Fe}$ Mössbauer spectra of such materials and on structure- 
property correlations, such as activity or selectivity with respect to the fraction or absolute content of a given doublet component.

For all the reasons discussed above, it appears obvious that the detailed analysis of the ${ }^{57} \mathrm{Fe}$ Mössbauer spectra of $\mathrm{Fe}-\mathrm{N}-\mathrm{C}$ materials cannot lean only on the experimental spectra of known macrocycles and that a complementary theoretical approach is required to interpret the Mössbauer spectra of such materials. No theoretical study has hitherto focused on predicting the two Mössbauer spectral parameters of $\mathrm{FeN}_{\mathrm{x}} \mathrm{C}_{\mathrm{y}}$ moieties in such materials. The first parameter, the isomer shift, $\delta$, is in the specific case of ${ }^{57} \mathrm{Fe}$ inversely proportional to the density of s-electrons at the absorbing nucleus. It can be computed from a molecular orbital method and reported relative to the isomer shift of a reference compound ${ }^{25-28}$. In this work, we focused our DFT efforts in calculating $\Delta E_{\mathrm{QS}}$, while methodologies to calculate $\delta$ were not investigated, due to the non-discriminating values of $\delta$ for the experimental doublets D1 and $\mathrm{D} 2$ in $\mathrm{Fe}-\mathrm{N}-\mathrm{C}$ catalysts. The second parameter, $\Delta E_{\mathrm{QS}}$, can be computed from the nuclear quadrupole moment and the electric field gradient at the absorbing ${ }^{57} \mathrm{Fe}$ nucleus. In recent years, calculation of the $\delta$ and $\Delta E_{\mathrm{QS}}$ values for several Fe coordination geometries have become straightforward with computer programs based on the Quantum Chemistry Density Functional Theory (DFT), using either localized molecular orbital (cluster) ${ }^{27-38}$ or periodic approaches $^{39-41}$. Despite the apparent easiness of calculating values of $\delta$ and $\Delta E_{\mathrm{QS}}$, the use of DFT as a tool for experimental spectral interpretation is hampered by the strong dependence of the calculated values for $\delta$ and, in particular, for $\Delta E_{\mathrm{QS}}$ on (i) the DFT exchange-correlation (XC) functional and (ii) the chosen parameterized set of wave functions (basis set) ${ }^{29-38,40}$. Recent DFT studies proposed different combinations of functionals and bases in order to achieve good agreement between experimental and computed Mössbauer parameters in ferrocene $^{29}$, Fe-porphyrin ${ }^{32}, \beta$-diketiminate ${ }^{31}$ derivatives and other iron complexes. ${ }^{33,35-36,38}$ Failures of DFT to reproduce the experimental $\Delta E_{\mathrm{QS}}$ values were reported for other compounds. ${ }^{33-35,38}$ A study on computed results obtained for a unique Fe coordination but resorting to eight different $\mathrm{XC}$ functionals and two basis sets revealed that the mean error between computed and experimental $\Delta E_{\mathrm{QS}}$ was $0.2 \mathrm{~mm} \cdot \mathrm{s}^{-1}$ for $\Delta E_{\mathrm{QS}}<2 \mathrm{~mm} \cdot \mathrm{s}^{-1}$, and $0.4 \mathrm{~mm} \cdot \mathrm{s}^{-}$ ${ }^{1}$ for $\Delta E_{\mathrm{QS}}>2 \mathrm{~mm} \cdot \mathrm{s}^{-1}$, excluding obvious outliers cases ${ }^{30}$. While no particular issues arise with zero-spin iron entities $(S=0)$, more difficulties may be found in the case of degenerate ground states, or closely spaced low-lying energy states of medium $(S=1)$ or high $\operatorname{spin}(S=2)$ iron complexes with square-planar coordination ${ }^{30,33}$. Other sources of error that have been pointed 
out are, e.g. the adequacy of the geometrical model or the quality of the optimized structures as cluster or extended periodic structures. ${ }^{31,33,40}$

The first object of this study is to explore the ability of DFT methodologies to predict $\Delta E_{\mathrm{QS}}$ values for a range of hypothetical $\mathrm{FeN}_{4}$ structures with different cluster size, metal-oxidation and spin state. For this purpose, the two most accepted sub-families of ORR-active $\mathrm{FeN}_{4}$ moieties present in pyrolyzed $\mathrm{Fe}-\mathrm{N}-\mathrm{C}$ materials were modeled, namely the $\mathrm{FeN}_{4} \mathrm{C}_{10}$ and $\mathrm{FeN}_{4} \mathrm{C}_{12}$ sub-groups ${ }^{10,22,42}$. The results of this theoretical study are then combined with the experimental Mössbauer data recorded for a well-defined Fe-N-C catalyst containing only atomically-dispersed iron species. To better discriminate the spin state and oxidation state of iron moieties, the catalyst was characterized also by Mössbauer spectroscopy in the presence of an external magnetic field, superconducting quantum interference device (SQUID) and electron paramagnetic resonance (EPR).

Mössbauer spectroscopy in the presence of an external magnetic field unambiguously reveals the major presence of low-spin ferrous centers accompanied by high-spin ferric centers in the Fe-N-C catalyst. The presence of high-spin ferric centers is independently supported by EPR measurements. Combined with the theoretical insights, we are able to conclude that the identified high-spin ferric signal can be assigned to $\mathrm{Fe}(\mathrm{III}) \mathrm{N}_{4} \mathrm{C}_{12}$ porphyrinic moieties, corresponding to the experimental doublet D1 $\left(\Delta E_{\mathrm{QS}}\right.$ in the range $\left.0.6-1.3 \mathrm{~mm} \cdot \mathrm{s}^{-1}\right)$. The lowspin ferrous signal is assigned to $\mathrm{Fe}(\mathrm{II}) \mathrm{N}_{4} \mathrm{C}_{10}$ pyridinic moieties, corresponding to the experimental doublet $\mathrm{D} 2\left(\Delta E_{\mathrm{QS}}\right.$ in the range $\left.1.7-3.1 \mathrm{~mm} \cdot \mathrm{s}^{-1}\right)$. A minor fraction of the Mössbauer signal with $\Delta E_{\mathrm{QS}}$ values falling in the intermediate region situated between 1.3 and $1.7 \mathrm{~mm} \cdot \mathrm{s}^{-1}$ is ambiguous and may be assigned either to high-spin ferric moieties $\left(\mathrm{Fe}(\mathrm{III}) \mathrm{N}_{4} \mathrm{C}_{10}\right.$ ) or low-spin ferrous moieties $\left(\mathrm{Fe}(\mathrm{II}) \mathrm{N}_{4} \mathrm{C}_{12}\right.$ ). The combination of the present DFT study with experimental ${ }^{57} \mathrm{Fe}$ Mössbauer spectroscopy under external magnetic field and other independent methods therefore leads to a detailed and rational interpretation of the spectroscopic signatures and electronic states of $\mathrm{FeN}_{\mathrm{x}} \mathrm{C}_{\mathrm{y}}$ moieties in $\mathrm{Fe}-\mathrm{N}-\mathrm{C}$ materials. 


\section{Computational and Experimental Methods}

\subsection{Computational Method}

DFT calculations were carried out with the cluster and periodic approaches using, respectively, deMon $2 \mathrm{k}^{43}$ and VASP ${ }^{44-47}$ computer programs on graphene sheets integrating various moieties from the $\mathrm{FeN}_{4} \mathrm{C}_{10}$ and $\mathrm{FeN}_{4} \mathrm{C}_{12}$ sub-groups (Figure 1).

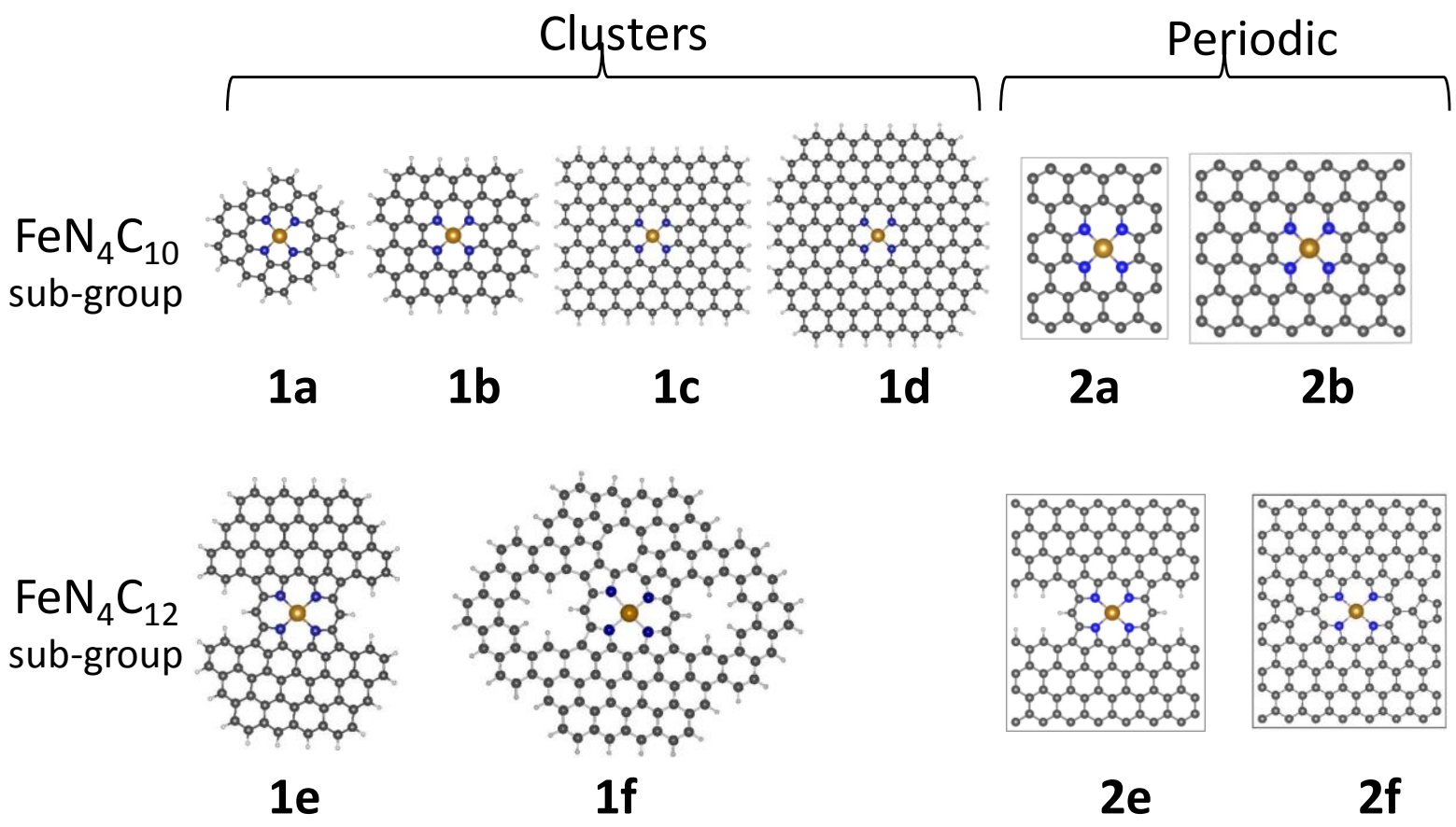

Figure 1.Cluster and periodic ferrous models of $\mathrm{FeN}_{4} \mathrm{C}_{\mathrm{y}}(\mathrm{y}=10$ or 12) moieties considered in this work as representative Fe sites in Fe-N-C materials. The optimized structures at PBE/DZVP2 (1a-1f) and PBE-PBC $(2 \mathrm{a}, \mathrm{b}, \mathrm{e}, \mathrm{f})$ level of theory are presented. See text for the differences in the optimized geometry parameters with the other methods. The atomic color code is the following: brown for Fe; blue for N; dark grey for C, light grey for $\mathrm{H}$.

The ferrous $\mathrm{FeN}_{4} \mathrm{C}_{10}$ and $\mathrm{FeN}_{4} \mathrm{C}_{12}$ clusters are modeled, respectively, with $1 \mathrm{a}$ - $1 \mathbf{d}$ and $\mathbf{1 e}-\mathbf{1 f}$ in Figure 1. The dangling bonds in all clusters and also in the periodic $2 \mathrm{e}$ system were saturated with hydrogen atoms. To model the respective ferric moieties, $\mathrm{OH}^{-}$is adsorbed in end-on mode on the ferric site in order to preserve the electric-charge neutrality. The resulting $\mathrm{OH}^{-} \mathrm{Fe}(\mathrm{III}) \mathrm{N}_{4} \mathrm{C}_{\mathrm{y}}$ moieties (labeled e.g. as 1a-OH) are exemplified in Figure S1. The electrons in the $\mathrm{C}, \mathrm{N}$ and $\mathrm{H}$ atoms are described by triple- $\zeta$ basis sets ${ }^{48}$. For the iron electrons we examined the performance of the double- $\zeta$ plus polarization ${ }^{48}$ and the original Wachter's ${ }^{49}$ bases, contracted to $(62111111 / 331211 / 3111)$. Three generalized gradient corrected (GGA) exchange-correlation functionals were tested: (1) the revised Perdew-Burke-Ernzerhof's 
exchange $^{50-51}$ and the Lee, Yang and Parr correlation ${ }^{52}$ (rPBE-LYP); (2) the Perdew-Burke-Ernzerhof's exchange-correlation (PBE) and (3) the OPTX exchange of Handy and Cohen ${ }^{53}$ coupled to the Lee, Yang and Parr correlation ${ }^{52}$ (OLYP). The geometries of the clusters 1a and 1e were fully optimized at every level of theory. Larger models (clusters 1b-1d, 1f) up to the size of the experimental graphitic crystallites in high surface-area Fe-N-C materials $(\sim 2 \mathrm{~nm})$ were fully optimized with rPBE-LYP/DZVP2, PBE/DZVP2 and PBE/Wachter's. No symmetry constraints were imposed. In deMon2k code, automatically generated auxiliary functions up to $\mathrm{l}=2$ (for the metal atom) and 3 (for $\mathrm{H}, \mathrm{C}$ and $\mathrm{O}$ atoms) were used for fitting the density with the GGA functionals. ${ }^{54}$ The GGA functionals were also coupled to an empirical dispersion (D) term. ${ }^{55}$ A quasi-Newton method in internal redundant coordinates with analytical energy gradients was used for structure optimization. For the numerical integrations of the XC energy and potential, we used an adaptive grid with tighten threshold $\left(10^{-8}\right.$ a.u. $){ }^{56}$ The convergence was based on the Cartesian gradient and displacement vectors with thresholds of $10^{-3}$ a.u. and the energy convergence was set to $10^{-7}$ a.u.

DFT calculations with periodic boundary conditions, carried out with VASP code, used the models 1a and 1e to build the unit cells. In the case of model 1a, two unit cells were considered; one with the size of $9.94 \AA$ x $12.64 \AA$ and the other $14.78 \AA$ x $12.80 \AA$ (models $\mathbf{2 a}$ and $\mathbf{2} \mathbf{b}$ in Figure 1, respectively). In the case of the cluster model $\mathbf{1 e}$, the corresponding periodic structure was modeled using either a cell size $17.18 \AA$ x $20.87 \AA$ (model 2e) or 17.17 $\AA$ x $21.0 \AA$ (model 2f). Vacuum region of $15 \AA$ was introduced in the $z$-direction in order to eliminate interactions between the graphene sheet and its periodic images. All the DFT calculations with periodic boundary conditions (PBC) were performed using generalized gradient approximation (GGA) with PBE exchange-correlation functional ${ }^{50}$ and VASP 5.2 recommended GW projector augmented-wave pseudopotentials. ${ }^{57-58}$ For Fe pseudopotential that treats $3 s$ and $3 p$ states as valence states was used. Electric field gradients at the positions of the Fe nuclei were calculated using the method reported in reference ${ }^{39}$ as implemented in VASP. To obtain electric field gradients cut-off energy for the plane wave basis set of 800 $\mathrm{eV}$, break condition for electronic SC-loop of $10^{-6} \mathrm{eV}$ and $8 \times 8 \times 1$ gamma centered mesh were used for the model $\mathbf{2 a}$ and $4 \times 4 \times 1$ mesh for the model $\mathbf{2 b}$ and $\mathbf{2 e - 2 f}$. In all cases, the FermiDirac smearing method with sigma set to 0.03 was used. In addition, all the calculations included support grid for the evaluation of the augmentation charges. 
The quadrupole splitting energy is computed as the coupling between the nuclear quadrupole moment $(Q)$ of the non-spherical nucleus and the principal components $V_{\mathrm{ii}}(i=x, y, z)$ of the electric field gradient (EFG) tensor at ${ }^{57} \mathrm{Fe}$ nucleus using the equation:

$\Delta E_{Q}=\frac{1}{2} e Q V_{z z} \sqrt{1+\frac{\eta^{2}}{3}}$

In the $\Delta E_{\mathrm{Q}}$ equation, $e$ is the charge of the electron and the asymmetry parameter $\eta$ is computed as $\eta=\left(V_{x x}-V_{y y}\right) / V_{z z}$, where $\left|V_{z z}\right| \geq\left|V_{y y}\right| \geq V_{x x}$. The nuclear quadrupole moment, $Q$, for the $J=3 / 2$ state is taken to be 0.16 barn. Computation of $\Delta E_{Q}$ and $\eta$ therefore becomes a question of computing the EFG tensor, which is readily obtained as an expectation value of the EFG operator, $V_{i j}=\left\langle\Psi_{0}\left|\frac{3 i j-r^{2}}{r^{5}}\right| \Psi_{0}\right\rangle$, for the electronic ground state $\Psi_{0}$ and $i, j=$ $x, y, z$ being the components of the electron radius vector $\boldsymbol{r}$. In the remainder, the calculated value of $\Delta E_{\mathrm{Q}}$ are reported in units of $\mathrm{mm} \cdot \mathrm{s}^{-1}$ (labeled as $\Delta E_{\mathrm{QS}}$ ), for a direct comparison to experimentally reported values.

\subsection{Experimental Methods}

\section{Synthesis of the $\mathrm{Fe}_{0.5}-\mathrm{NC}$ catalyst}

The Fe-N-C catalyst, labelled $\mathrm{Fe}_{0.5}-\mathrm{NC}$, was prepared from $\mathrm{Fe}^{\mathrm{II}}$ acetate, 1,10-phenanthroline and a $\mathrm{Zn}^{\mathrm{II}}$ zeolitic imidazolate framework (ZIF-8 with formula $\mathrm{ZnN}_{4} \mathrm{C}_{8} \mathrm{H}_{12}$, purchased from BASF under trademark Basolite Z1200). One gram of a catalyst precursor containing $0.5 \mathrm{wt} \%$ Fe with a mass-ratio phen/ZIF-8 of 20/80 was prepared by dry ball-milling. The milling was carried out in a $\mathrm{ZrO}_{2}$ crucible with $100 \mathrm{ZrO}_{2}$ balls of $5 \mathrm{~mm}$ diameter using a planetary ballmiller (FRITSCH Pulverisette 7 Premium) for 4 cycles of $30 \mathrm{~min}$ at $400 \mathrm{rpm}$. The resulting catalyst precursor was then pyrolyzed at $1050{ }^{\circ} \mathrm{C}$ in Ar for $1 \mathrm{~h}$, leading to $\mathrm{Fe}_{0.5}-\mathrm{NC}$. This synthesis has been previously shown by Mössbauer spectroscopy and XANES-EXAFS to result in an ORR-active catalyst that comprise only atomically-dispersed $\mathrm{FeN}_{\mathrm{x}} \mathrm{C}_{\mathrm{y}}$ moieties. ${ }^{10}$ A reference sample free of iron or only with trace iron, labelled NC, was prepared identically as $\mathrm{Fe}_{0.5}-\mathrm{NC}$ except that no $\mathrm{Fe}^{\mathrm{II}}$ acetate was added in the catalyst precursor.

\section{Measurement of the Mössbauer spectra}

${ }^{57} \mathrm{Fe}$ Mössbauer spectra of $\mathrm{Fe}_{0.5}$-NC were measured with a ${ }^{57} \mathrm{Co}: \mathrm{Rh}$ source kept at ambient temperature $(294 \mathrm{~K})$. Spectra in the absence of external magnetic field were collected with the 
absorber at variable temperatures in the range 5.5-296 K using a Janis CCR $5 \mathrm{~K}$ cryostat. Additional measurements were performed in the presence of an external magnetic field applied either parallel or perpendicular to the $\gamma$-rays with the absorber at $4.5 \mathrm{~K}$ on a strongfield Mössbauer spectrometer equipped with an Oxford Instruments Spectromag 4000 cryostat containing an $8 \mathrm{~T}$ split-pair superconducting magnet. Both spectrometers were operated with a triangular velocity waveform, in a constant acceleration mode in transmission geometry, and were calibrated at $295 \mathrm{~K}$ with an $\alpha$-iron foil. The isomer shift scale is referred to metallic $\alpha-\mathrm{Fe}$ at room temperature.

\section{Analysis of the Mössbauer spectra}

The broadened quadrupole components of the Mössbauer spectra measured in the absence of a magnetic field were fitted with appropriate superposition of Lorentzian lines (fixed linewidth of $0.25 \mathrm{~mm} \cdot \mathrm{s}^{-1}$ ), assuming Gaussian distributions of the electric quadrupole interaction for each spectral component. Up to three independent components were sometimes necessary to fit coherently the series of spectra acquired on $\mathrm{Fe}_{0.5}-\mathrm{NC}$ at different temperatures.

In order to fit the complex experimental spectra observed in the presence of an external magnetic field (shown later), simple histogram distributions of magnetic sextets with hyperfine fields up to $50 \mathrm{~T}$ were employed. Mössbauer spectra of the same Fe-N-C sample measured with a magnetic field applied parallel or perpendicular to the gamma rays were fitted simultaneously with a single distribution of hyperfine fields. For each hyperfine field considered in the distribution, the only difference being theoretically expected when changing from a parallel to a perpendicular external magnetic field is the relative intensity of the six Mössbauer lines, which is 3:0:1:1:0:3 or 3:4:1:1:4:3, respectively, for the lines 1 to 6 , as labelled from the lowest to the highest position of the lines on the $\mathrm{x}$-axis velocity range of Mössbauer spectra. Therefore, during the fitting, only the relative intensity of the lines 2 and 5 were fitted as a function of the direction of the external magnetic field, since it is known that the relative intensity of the lines 1, 3, 4 and 6 do not vary with the direction of the external magnetic field. As will be shown later, this fitting approach can however not result in a perfect fit in all regions of the spectra, since species characterized by low magnetic fields and high quadrupole splittings, the quadrupole interaction cannot be physically treated as a perturbation of the Zeeman magnetic interaction, and thus the Mössbauer components cannot be rigorously fitted simply using magnetic sextets. ${ }^{59}$

\section{SQUID characterisation}


The molar magnetic susceptibility $\chi_{\mathrm{m}}$ was measured from 2 to $300 \mathrm{~K}$ for $\mathrm{Fe}_{0.5}$ - $\mathrm{NC}$ and $\mathrm{NC}$ with a Superconducting QUantum Interference Device (SQUID) (MPMS XL-7T, Quantum Design) at a magnetic field of 5,000 Oe. A mass of $16 \mathrm{mg}$ of powder sample was introduced in a polymer straw. The average effective magnetic moment of iron atoms $\left(\mu_{\text {eff }}\right)$ was then obtained by fitting the plot of $1 / \chi_{m}$ (in mol of iron atoms per emu) vs. $1 / \mathrm{T}$ with a linear law.

\section{Electron paramagnetic resonance (EPR)}

EPR spectra were acquired at $9.65 \mathrm{GHz}$. A few mg of the sample $\mathrm{Fe}_{0.5}-\mathrm{NC}$ was introduced in a glass capillary. The spectra were acquired at $10 \mathrm{~K}$ for different power/amplitude ratios. The optimum power and amplitude were found to be $3 \mathrm{~mW}$ and $0.3 \mathrm{mT}$, respectively.

\section{Results}

\subsection{Theoretical study}

We first discuss the impact of various exchange correlation functionals and basis sets on calculated values of $\Delta E_{\mathrm{QS}}$. This analysis is carried out for two ferrous moieties representative for the $\mathrm{FeN}_{4} \mathrm{C}_{10}$ and $\mathrm{FeN}_{4} \mathrm{C}_{12}$ sub-groups. Subsequently, the effects of the cluster size and iron oxidation state on $\Delta E_{\mathrm{QS}}$ are investigated and discussed.

\section{Effect of exchange-correlation functionals and basis sets on calculated values of $\Delta E_{\mathrm{QS}}$}

In this section, we focus on the smallest $\mathbf{1 a}$ and $\mathbf{1 e}$ clusters and their periodic $\mathbf{2 a}$ and $\mathbf{2 e , f}$ analogs, with $\mathrm{Fe}$ in oxidation state II, in low or medium spin state. The high spin state $\mathrm{S}=2$ was not considered since it is well known to result in an isomer shift $\geq 0.9 \mathrm{~mm} \cdot \mathrm{s}^{-1},{ }^{60}$ a value that has never been unambiguously experimentally observed in pyrolyzed Fe-N-C catalysts hitherto ${ }^{6}$. Note that the geometries were optimized without imposing any broken $\mathrm{D}_{4 \mathrm{~h}}$ symmetry. ${ }^{10}$ All the DFT methods reveal that the electronic state with spin $\mathrm{S}=0$ is slightly less favored from an energy viewpoint, as follows from the positive values of the relative energy, $\Delta E_{S=0}$, in Table 1 . In the cluster calculations of $\Delta E_{\mathrm{QS}}$, the closed-shell electronic structures $(S=0)$ have a relatively low sensitivity toward a particular DFT method. For the 1a cluster, $\Delta E_{\mathrm{QS}}$ is in the range $2.4-3.1 \mathrm{~mm} \cdot \mathrm{s}^{-1}$ (Table 1, row No. 1-6) and for cluster 1e in the range 1.6-2.0 $\mathrm{mm} \cdot \mathrm{s}^{-1}$ (row No. 8-13). The differences between $\Delta E_{\mathrm{QS}}$ values obtained with the three functionals for a given basis set is within $0.4 \mathrm{~mm} \cdot \mathrm{s}^{-1}$, and between the two bases for a given functional is within $0.3 \mathrm{~mm} \cdot \mathrm{s}^{-1}$. Accepting that differences of $0.4 \mathrm{~mm} \cdot \mathrm{s}^{-1}$ for $\Delta E_{\mathrm{QS}} \geq 2$ 
$\mathrm{mm} \cdot \mathrm{s}^{-1}$ and $0.2 \mathrm{~mm} \cdot \mathrm{s}^{-1}$ for $\Delta E_{\mathrm{QS}} \leq 2 \mathrm{~mm} \cdot \mathrm{s}^{-1}$ are within the computational error, ${ }^{30}$ we conclude on a good agreement between the different methods in calculated $\Delta E_{\mathrm{QS}}$ values for the low-spin clusters. A good agreement is also observed between the low-spin cluster and periodic (PBE/PBC) approaches (e.g. for structure $\mathbf{2 a}$, compare row No. 7 to rows 1-6).

In contrast, for the open-shell cluster structures $(\mathrm{S}=1)$, the distribution of $\Delta E_{\mathrm{QS}}$ values is significantly larger. In the 1a cluster, $\Delta E_{\mathrm{QS}}$ varies between 1.5 and $3.3 \mathrm{~mm} \cdot \mathrm{s}^{-1}$ (Table 1 , row No. 1-6) while $\Delta E_{\mathrm{QS}}$ in the corresponding periodic structure $\mathbf{2 a}$ is $3.3 \mathrm{~mm} \cdot \mathrm{s}^{-1}$ (row 7). In the medium-spin 1e cluster $\left(\mathrm{FeN}_{4} \mathrm{C}_{12}\right), \Delta E_{\mathrm{QS}}$ varies between 0.1 and $1.6 \mathrm{~mm} \cdot \mathrm{s}^{-1}$ (rows 8-13) while $\Delta E_{\mathrm{QS}}$ in the periodic structures $\mathbf{2 e - 2 \mathrm { f }}$ is $1.8 \mathrm{~mm} \cdot \mathrm{s}^{-1}$ (rows 14-15). The main outliers are however obtained with rPBE-LYP functionals (rows 1-2; 8-9), whereas the other two functionals (PBE and OLYP) result in much closer $\Delta E_{\mathrm{QS}}$ values (row No. 3-6 and 10-13). The PBE and OLYP functionals with the DZVP2 basis set result in an excellent agreement of $\Delta E_{\mathrm{QS}}$ with the periodic approach, in the range of $2.9-3.3 \mathrm{~mm} \cdot \mathrm{s}^{-1}$ for models $1 \mathrm{a}$ and $2 \mathrm{a}$ (row No. 3, 5, 7) and in the range of 1.6-1.8 $\mathrm{mm} \cdot \mathrm{s}^{-1}$ for models $\mathbf{1 e}$ and $\mathbf{2 e - 2 f}$ (row No. 10, 12, 1415). 
Table 1. Quadrupole splitting energy $\Delta E_{\mathrm{QS}}$ (in $\mathrm{mm} \cdot \mathrm{s}^{-1}$ ) and relative energy $\Delta E$ (in $\mathrm{eV}$ ) computed with three DFTGGA functionals and two basis sets for $\mathrm{Fe}(\mathrm{II}) \mathrm{N}_{4} \mathrm{C}_{10}$ cluster(1a) and periodic (2a) models, and $\mathrm{Fe}(\mathrm{II}) \mathrm{N}_{4} \mathrm{C}_{12}$ cluster (1e) and periodic (2e and 2f) models. The models structures are shown in Figure 1. Low ( $\mathrm{S}=0)$ and medium $(\mathrm{S}=1)$ spin states are considered. The relative energies are computed from the total energies $\left(E_{\mathrm{tot}}\right)$ as $\Delta E_{S=0}=$ $E_{\mathrm{tot}}(\mathrm{S}=0)-E_{\mathrm{tot}}(\mathrm{S}=1)$.

\begin{tabular}{|c|c|c|c|c|c|}
\hline Row No. & Functional / Basis set & Model & $\begin{array}{c}\Delta E_{\mathrm{QS}} \\
(\mathrm{S}=0)\end{array}$ & $\begin{array}{c}\Delta E_{\mathrm{QS}} \\
(\mathrm{S}=1)\end{array}$ & $\Delta E_{\mathrm{S}=0}$ \\
\hline 1 & rPBE-LYP / DZVP2 & $1 \mathrm{a}$ & 2.7 & 2.2 & 0.38 \\
\hline 2 & rPBE-LYP / Wachter's & $1 \mathrm{a}$ & 2.4 & 1.5 & 0.41 \\
\hline 3 & PBE / DZVP2 & $1 \mathrm{a}$ & 2.8 & 2.9 & 0.64 \\
\hline 4 & PBE / Wachter's & $1 \mathrm{a}$ & 2.5 & 2.6 & 0.73 \\
\hline 5 & OLYP / DZVP2 & $1 \mathrm{a}$ & 3.1 & 3.3 & 0.65 \\
\hline 6 & OLYP / Wachter's & $1 \mathrm{a}$ & 2.8 & 3.1 & 0.89 \\
\hline 7 & PBE-PBC (VASP) & $2 \mathrm{a}$ & 2.4 & 3.3 & 0.63 \\
\hline 8 & rPBE-LYP / DZVP2 & $1 \mathrm{e}$ & 2.0 & 0.7 & 0.61 \\
\hline 9 & rPBE-LYP / Wachter's & $1 \mathrm{e}$ & 1.7 & 0.1 & 1.20 \\
\hline 10 & $\mathrm{PBE} / \mathrm{DZVP} 2$ & $1 \mathrm{e}$ & 1.6 & 1.6 & 0.73 \\
\hline 11 & PBE / Wachter's & $1 \mathrm{e}$ & 1.6 & 1.1 & 1.09 \\
\hline 12 & OLYP / DZVP2 & $1 \mathrm{e}$ & 1.6 & 1.6 & 1.16 \\
\hline 13 & OLYP / Wachter's & $1 \mathrm{e}$ & 1.6 & 1.2 & 1.24 \\
\hline 14 & PBE-PBC (VASP) & $2 \mathrm{e}$ & 1.3 & 1.8 & 0.86 \\
\hline 15 & PBE-PBC (VASP) & $2 f$ & 1.2 & 1.8 & 0.81 \\
\hline
\end{tabular}

The larger discrepancy in the $S=1$ state relative to low-spin state is not unexpected, since the EFG values depend on the ground-state electronic structure, namely on the occupation of the $3 d$ orbitals of $\mathrm{Fe}^{33}$. The large $\Delta E_{\mathrm{QS}}$ differences seen for DFT methods in the medium-spin states are often attributed to the capability of a XC functional to yield the true ground-state 
electronic structure in iron compounds ${ }^{29,32-33}$. When the symmetry is not imposed, as in our calculations, the degeneracy of $3 d$-spin-orbitals is removed and the occupation of the $3 d$ orbitals becomes highly dependent on the particular XC functional and on the chosen basis sets $^{9,12}$. In the present study, this general finding is evidenced through the comparison of the occupation of the $3 d$ orbitals below the Fermi level in cluster 1a and periodic $2 \mathbf{a}$ models (see Table S1). A significantly lower occupation of the $d z^{2}$ orbital in cluster 1a with rPBELYP/Wachters than that with all other functionals/basis sets is observed. On the other hand, these different $\mathrm{Fe}$ electronic states are in geometrically-equivalent structures (see supporting Tables S2-S3 and related text). Thus, we conclude that the larger discrepancy for $S=1$ spin state is due to the difficult description of the occupation of the $3 d$ orbitals, in agreement with previous theoretical studies ${ }^{29-38}$.

\section{Influence of cluster size on calculated values of $\Delta E_{\mathrm{QS}}$}

To gain understanding in the importance of the edge or in-plane location of the iron center in a graphene plane, we calculated $\Delta E_{\mathrm{QS}}$ for larger cluster sizes than those of structures $1 \mathrm{a}$ and 1e, up to $2.5 \mathrm{~nm}$ (models $\mathbf{1 b}, \mathbf{c}, \mathbf{d}$ and $\mathbf{1 f}$ in Figure 1). This value resembles the typical size of graphene crystallites in Fe-N-C materials ${ }^{61}$. Periodic boundary conditions were also imposed to the corresponding larger unit cells (models $\mathbf{2 b}$ and $\mathbf{2 f}$ in Figure 1) in order to evaluate the effect of the distance between two adjacent iron sites. The PBE functional was chosen because it performs similarly to OLYP and is also used in the periodic computations. The changes of $\Delta E_{\mathrm{QS}}$ values with cluster size are depicted in Figure 2 while the exact values are reported in Table S4. 

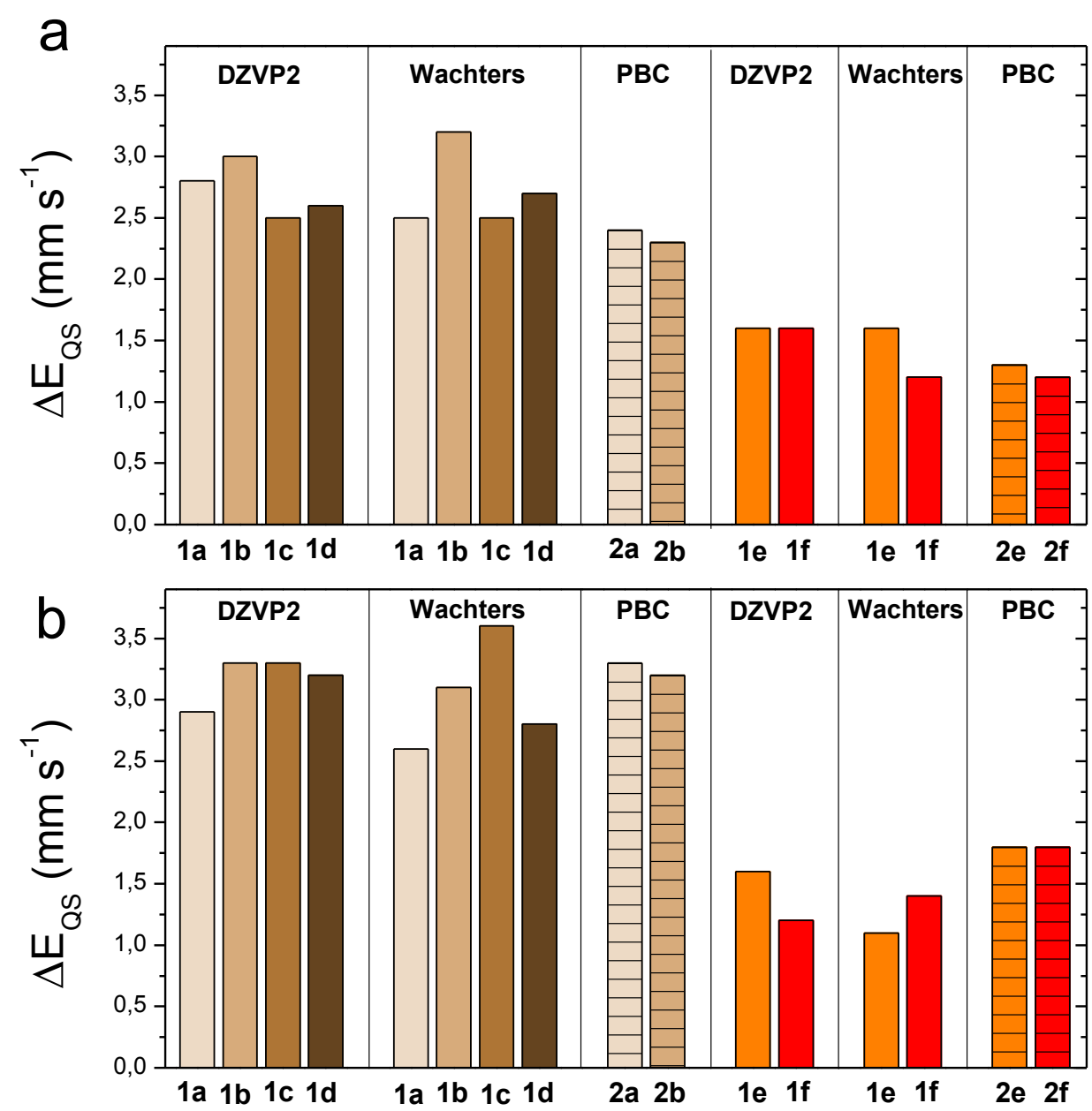

Figure 2. Quadrupole splitting energies, $\Delta E_{\mathrm{QS}}$, as a function of cluster size, 1a-1f, and unit cells, $\mathbf{2 a - 2 b}, \mathbf{2} \mathbf{f}$, of $\mathrm{FeN}_{4} \mathrm{C}_{10}$ and $\mathrm{FeN}_{4} \mathrm{C}_{12}$ ferrous models with low $(\mathrm{S}=0)$ spin (Fig. 2a) and medium $(\mathrm{S}=1)$ spin (Fig. 2b). The results are obtained with PBE functional in the periodic (PBC) and cluster (with DZVP2 or Wachters basis) approaches. Columns for periodic approaches are hatched.

The magnitude of changes in $\Delta E_{\mathrm{QS}}$ with cluster size is close to the computational error bar for the $\mathrm{FeN}_{4} \mathrm{C}_{10}\left(0.4 \mathrm{~mm} \cdot \mathrm{s}^{-1}\right)$ and $\mathrm{FeN}_{4} \mathrm{C}_{12}$ groups $\left(0.2 \mathrm{~mm} \cdot \mathrm{s}^{-1}\right)$, and only slightly above the error bar for few cases. This finding correlates well with the very small changes in the Fe-N bond distances and N-Fe-N bond angles in models 1a to 1d (Table S5). Indeed, the fluctuation of the bond distances with cluster size is restrained to a maximum of $0.05 \AA$ for all considered methods and spin states. Regarding bond angles, for $S=0$, the trends with cluster size from 1a to 1d are similar with the PBE/Wachters and PBE/DZVP2 methods, showing a reduced spreading of the four $\mathrm{N}_{\mathrm{i}}-\mathrm{Fe}-\mathrm{N}_{\mathrm{j}}$ bond angles relative to $90^{\circ}$. Thus, as the cluster size is increased, the $\mathrm{FeN}_{4}$ core becomes closer to a $\mathrm{D}_{4 \mathrm{~h}}$ symmetry group. We also note that $\mathrm{FeN}_{4} \mathrm{C}_{12}$ structures with hydrogen (2e) and without hydrogen (2f) in the third coordination sphere have 
a nearly identical $\Delta E_{\mathrm{QS}}$ (see Figure 2, two last columns). Therefore one may conclude that $\Delta E_{\mathrm{QS}}$ is mainly sensitive to the spatial arrangement of nitrogen and carbon atoms in the two first coordination spheres, while the ordering of atoms at longer distances negligibly impacts $\Delta E_{\mathrm{QS}}$.

\section{Effect of Fe oxidation state on calculated values of $\Delta E_{\mathrm{QS}}$}

Operando X-ray absorption spectroscopy studies systematically evidenced that pyrolyzed Fe$\mathrm{N}-\mathrm{C}$ catalysts contain a significant fraction of ferric moieties in their resting state, ${ }^{14}$ including the model $\mathrm{Fe}-\mathrm{N}-\mathrm{C}$ catalyst further experimentally characterized in the present study ${ }^{10}$. Therefore, ferric clusters were considered, and modeled by adsorbing an hydroxide anion to $\mathrm{Fe}(\mathrm{III})$. The models are presented in Figure S1, labeled $\mathbf{1 a - O H}$ and $\mathbf{1 d - O H}\left(\mathrm{FeN}_{4} \mathrm{C}_{10}\right)$ and $\mathbf{1 e -}$ $\mathbf{O H}$ and 1f-OH clusters $\left(\mathrm{FeN}_{4} \mathrm{C}_{12}\right)$. The quadrupole splitting energies are computed with the PBE and rPBE-LYP functionals. The latter was included because it resulted in the main outliers for the ferrous clusters 1a and 1e in medium spin (Table 1) and we wanted to examine its performance in the ferric compounds. Table 2 reports $\Delta E_{\mathrm{QS}}$ values and relative energies for these four clusters and two periodic models $(\mathbf{2 a - O H}$, and $\mathbf{2} \mathbf{e}-\mathbf{O H})$, at three different spin states. For each cluster, the agreement between the DFT methods is excellent (see rows 1-3; 4-5; 7-9; 10-11). The rPBE-LYP and PBE functionals, therefore, lead to similar results for ferric models, in contrast to the case with ferrous models. For the low-spin ferric structures, the three DFT methods agree well and give $\Delta E_{\mathrm{QS}}$ between $2.1-2.5 \mathrm{~mm} \cdot \mathrm{s}^{-1}$ in $\mathbf{1 a}-\mathbf{O H}, \mathbf{1 d - O H}$, $\mathbf{2 a - O H}\left(\mathrm{FeN}_{4} \mathrm{C}_{10}\right.$ sub-group) and $\Delta E_{\mathrm{QS}}$ between $1.8-2.1 \mathrm{~mm} \cdot \mathrm{s}^{-1}$ in $\mathbf{1 e - O H}, \mathbf{1 f}-\mathbf{O H}$ and $\mathbf{2 e - O H}$ ( $\mathrm{FeN}_{4} \mathrm{C}_{12}$ sub-group). However, the spread of the results is very large in the medium-spin state. $\Delta E_{\mathrm{QS}}$ values are between $0.9-2.5 \mathrm{~mm} \cdot \mathrm{s}^{-1}$ in $\mathbf{1 a - O H}, \mathbf{1 d - O H}, \mathbf{2 a - O H}$ and between 0.7-2.0 $\mathrm{mm} \cdot \mathrm{s}^{-1}$ in 1e-OH, $\mathbf{1 f}-\mathbf{O H}$ and $\mathbf{2 e - O H}$. In particular, the significant difference in the calculated $\Delta E_{\mathrm{QS}}$ value for the large $1 \mathbf{d}$ cluster $\left(2.5 \mathrm{or} 2.3 \mathrm{~mm} \cdot \mathrm{s}^{-1}\right)$ and the periodic $2 \mathrm{a}$ model $\left(1.1 \mathrm{~mm} \cdot \mathrm{s}^{-1}\right)$ is surprising. For the high-spin state, the agreement for the $\mathbf{1 a - O H}$ and $\mathbf{1 d - O H}$ clusters is excellent but a discrepancy again occurs with the periodic $\mathbf{2 a - O H}$ model. While more restrained compared to the case with the medium spin-state, some spreading of the results remains among 1e-OH, $\mathbf{1}-\mathbf{O H}$ and $\mathbf{2 e - O H}$ (porphyrinic sub-group), with $\Delta E_{\mathrm{QS}}$ values in the range of $0.6-1.1 \mathrm{~mm} \cdot \mathrm{s}^{-1}$. 
Table 2. Quadrupole splitting energy $\Delta E_{\mathrm{QS}}$ (in $\mathrm{mm} \cdot \mathrm{s}^{-1}$ ) and relative energy $\Delta E$ (in eV) computed with two DFTGGA functionals and two basis sets for $\mathrm{Fe}(\mathrm{III}) \mathrm{N}_{4} \mathrm{C}_{10}$ and $\mathrm{Fe}(\mathrm{III}) \mathrm{N}_{4} \mathrm{C}_{12}$ cluster and periodic models. The model structures are shown in Figure $\mathrm{S} 1$. Low $(\mathrm{S}=1 / 2)$, medium $(\mathrm{S}=3 / 2)$ and high $(\mathrm{S}=5 / 2)$ spin states are considered. The relative energies are computed from the total energies $\left(E_{\mathrm{tot}}\right)$ as $\Delta E_{n / 2}=E_{\mathrm{tot}}(\mathrm{S}=\mathrm{n} / 2)-E_{\mathrm{tot}}(\mathrm{S}=1 / 2)$ with $\mathrm{n}=3 / 2$ and $5 / 2$.

\begin{tabular}{|c|c|c|c|c|c|c|c|}
\hline $\begin{array}{l}\text { Row } \\
\text { No. }\end{array}$ & Functional / Basis set & Model & $\begin{array}{c}\Delta \mathrm{E}_{\mathrm{QS}} \\
(\mathrm{S}=1 / 2)\end{array}$ & $\begin{array}{c}\Delta \mathrm{E}_{\mathrm{QS}} \\
(\mathrm{S}=3 / 2)\end{array}$ & $\begin{array}{c}\Delta \mathrm{E}_{\mathrm{QS}} \\
(\mathrm{S}=5 / 2)\end{array}$ & $\Delta \mathrm{E}_{3 / 2}$ & $\Delta \mathrm{E}_{5 / 2}$ \\
\hline 1 & rPBE-LYP / DZVP2 & 1a-OH & 2.2 & 1.3 & 1.5 & 0.41 & 1.14 \\
\hline 2 & PBE / DZVP2 & 1a-OH & 2.3 & 0.9 & 1.4 & 0.28 & 1.05 \\
\hline 3 & PBE / Wachter's & 1a-OH & 2.1 & 0.9 & 1.4 & 0.83 & 0.96 \\
\hline 4 & PBE / DZVP2 & 1d-OH & 2.3 & 2.5 & 1.6 & 0.67 & 1.27 \\
\hline 5 & PBE / Wachter's & 1d-OH & 2.1 & 2.3 & 1.4 & 0.31 & 1.12 \\
\hline 6 & PBE-PBC (VASP) & 2a-OH & 2.5 & 1.1 & 0.7 & 0.30 & 0.70 \\
\hline 7 & rPBE-LYP / DZVP2 & 1e-OH & 1.9 & 0.8 & 0.8 & 0.24 & 0.60 \\
\hline 8 & $\mathrm{PBE} / \mathrm{DZVP} 2$ & 1e-OH & 1.9 & 0.7 & 0.6 & 0.19 & 0.52 \\
\hline 9 & PBE / Wachter's & 1e-OH & 2.1 & 0.8 & 0.7 & 0.12 & 0.45 \\
\hline 10 & PBE / DZVP2 & 1f-OH & 1.8 & 1.9 & 1.1 & -0.03 & 0.14 \\
\hline 11 & PBE / Wachter's & 1f-OH & 2.1 & 2.0 & 1.1 & 0.02 & 0.21 \\
\hline 12 & PBE-PBC (VASP) & $2 \mathrm{e}-\mathrm{OH}$ & 2.0 & 0.9 & 0.7 & 0.01 & 0.18 \\
\hline
\end{tabular}

We therefore examined if $\mathrm{OH}^{-}$adsorption led to distinct geometric changes as a function of the structural models and functional/basis set chosen, in particular for the ferric models in medium spin state. It is first noted that $\mathrm{OH}^{-}$adsorption on Fe leads to a non-planar geometry. In all cases, $\mathrm{Fe}(\mathrm{III})$ is situated above the graphene plane by 0.2-0.3 $\AA$ (calculated, but not reported in Tables). The geometric parameters of the ferric $\mathrm{FeN}_{4} \mathrm{C}_{10}$ and $\mathrm{FeN}_{4} \mathrm{C}_{12}$ groups are reported in Tables S6 and S7, respectively. While the spreading of the Fe-N bond distances and $\mathrm{N}-\mathrm{Fe}-\mathrm{N}$ angles in the ferric models is larger than in the corresponding ferrous models, the structural disorder caused by the $\mathrm{OH}$ adsorption is comparable for all models (see for example 
1a-OH and 1d-OH in medium spin state in Table S6), Thus, the large $\Delta E_{\mathrm{QS}}$ differences obtained for 1a-OH and 1d-OH on one hand, and 1e-OH and 1f-OH on the other hand, cannot be attributed to the slight structural changes induced by $\mathrm{OH}$-adsorption. In contrast, there might be a distinct $\mathrm{OH}$ orientation, depending on the model and spin-state, which could influence the electric field gradient components at the iron nucleus. Analyzing the minimum energy ferric structures, we could distinguish different $\mathrm{OH}$ orientations with respect to the $\mathrm{N}$ Fe-N plane. This can be quantified through the dihedral $\mathrm{H}-\mathrm{O}-\mathrm{Fe}-\mathrm{N}$ angle, $\theta$, which defines the angle between the $\mathrm{H}-\mathrm{O}-\mathrm{Fe}$ and the $\mathrm{N}-\mathrm{Fe}-\mathrm{N}$ planes (Figure $\mathrm{S} 2$ ). For O-H pointing above the $\mathrm{N}$ atom, the $\mathrm{H}-\mathrm{O}-\mathrm{Fe}-\mathrm{N}$ dihedral angle is $0^{\circ}$ and for $\mathrm{O}-\mathrm{H}$ pointing above the $\mathrm{N}-\mathrm{Fe}-\mathrm{N}$ bisector, the $\mathrm{H}-\mathrm{O}-\mathrm{Fe}-\mathrm{N}$ dihedral angle is $\sim 45^{\circ}$, as exemplified in Figure $\mathrm{S} 2$. The calculated $\theta$ values are summarized in Tables S6, S7.

For the ferric $\mathrm{FeN}_{4} \mathrm{C}_{10}$ clusters in low-spin, $\theta$ varies only between $8-11^{\circ}$, in line with the similar $\Delta E_{\mathrm{QS}}$ values calculated for all those structures. In contrast, in medium spin a large difference is observed for $\mathbf{1 a - O H}$ and $\mathbf{1 d - O H}$ models. The $\theta$ values are between $27^{\circ}-33^{\circ}$ in $\mathbf{1 a - O H}$ and $9^{\circ}$ in 1d-OH. The comparison with trends for $\Delta E_{\mathrm{QS}}$ values evidences a clear correlation between the $\theta$ and $\Delta E_{\mathrm{QS}}$ values for these medium-spin ferric models. For the high spin models, $\theta$ spans between 17 and $34^{\circ}$. We therefore identify an inverse correlation between $\theta$ and $\Delta E_{\mathrm{QS}}$ for $\mathrm{FeN}_{4} \mathrm{C}_{10}$ models in any spin state. Structures with $\theta$ values between $8-11^{\circ}$ have high $\Delta E_{\mathrm{QS}}$ in the range $2.0-2.5 \mathrm{~mm} \cdot \mathrm{s}^{-1}$, whereas those with larger $\theta$ have low $\Delta E_{\mathrm{QS}}$ in the range $0.9-1.6 \mathrm{~mm} \cdot \mathrm{s}^{-1}$.

A correlation between $\mathrm{OH}$ orientation and $\Delta E_{\mathrm{QS}}$ is established also for the porphyrinic $\mathrm{FeN}_{4} \mathrm{C}_{12}$ moieties (Table S7). In this case, the correlation between $\theta$ and $\Delta E_{\mathrm{QS}}$ is seemingly inversed. The structures with low $\theta\left(\sim 1^{\circ}\right.$ in $\mathbf{1 e - O H}$, medium- and high-spin states $)$ have low $\Delta E_{\mathrm{QS}}$ values between $0.6-0.8 \mathrm{~mm} \cdot \mathrm{s}^{-1}$ and the structures with high $\theta\left(\sim 9-21^{\circ}\right.$ in $\mathbf{1 e - O H}$, low spin, and in 1f-OH in all spin states) have high $\Delta E_{\mathrm{QS}}$ values in the range $1.1-2.1 \mathrm{~mm} \cdot \mathrm{s}^{-1}$. 


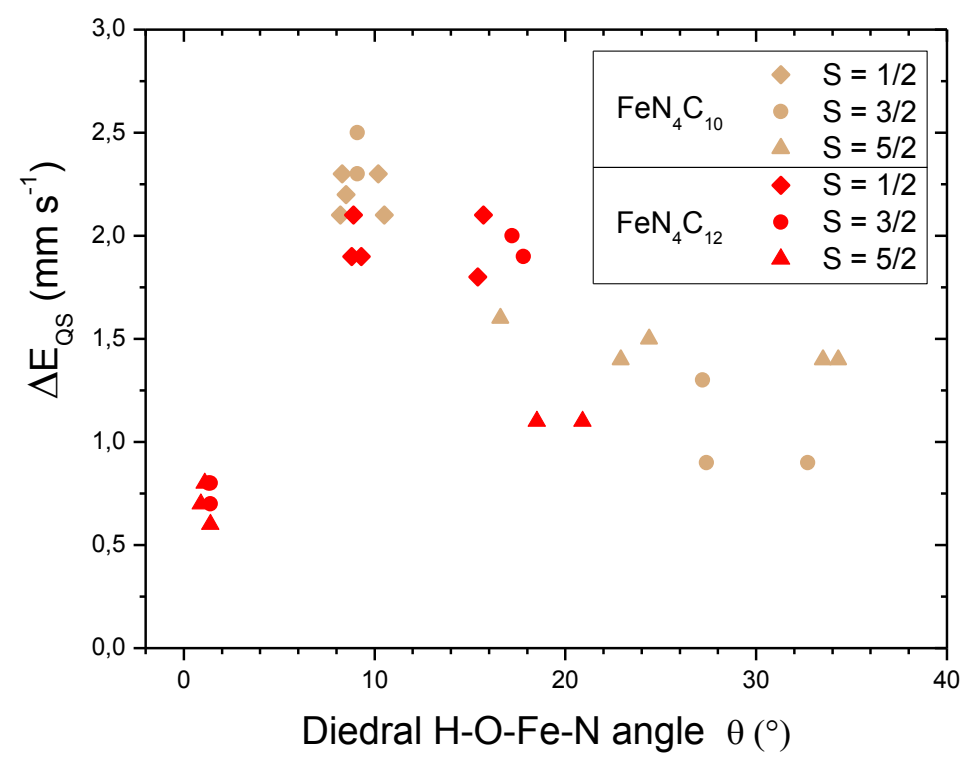

Figure 3. Calculated $\Delta \mathrm{E}_{\mathrm{QS}}$ values for ferric moieties as a function of the dihedral $\mathrm{O}-\mathrm{H}-\mathrm{Fe}-\mathrm{N}$ angle $(\theta)$ for the low, medium and high spin-states in the ferric $\mathrm{FeN}_{4} \mathrm{C}_{10}(\mathbf{1 a - O H}$ and $\mathbf{1 d - O H})$ and $\mathrm{FeN}_{4} \mathrm{C}_{12}(\mathbf{1 e - O H}$ and $\mathbf{1 f - O H})$ cluster models. The rPBE-LYP / DZVP2, PBE/DZVP2 and PBE/Wachters methods were used for cluster models, and PBE/PBC used for periodic models.

The trend of $\Delta E_{\mathrm{QS}}$ as a function of $\mathrm{OH}$ orientation for the three spin-states and all considered ferric structures is presented in Figure 3. Three distinct regions appear: (1) for $\theta \approx 1, \Delta E_{\mathrm{QS}}<1$ $\mathrm{mm} \cdot \mathrm{s}^{-1}$; (2) for $\sim 8<\theta<\sim 12^{\circ}, \Delta E_{\mathrm{QS}}>1.8 \mathrm{~mm} \cdot \mathrm{s}^{-1}$ and (3) for $\theta>\sim 12^{\circ}, \Delta E_{\mathrm{QS}}$ linearly decreases from $\sim 2$ to $1 \mathrm{~mm} \cdot \mathrm{s}^{-1}$ with increasing $\theta$ from $12^{\circ}$ to $35^{\circ}$. This graph unifies all the results for the ferric $\mathrm{FeN}_{4} \mathrm{C}_{10}$ and $\mathrm{FeN}_{4} \mathrm{C}_{12}$ models, demonstrating that the calculated $\Delta E_{\mathrm{QS}}$ value rather depends on the orientation of the $\mathrm{OH}$ group, and not much on the model geometry or spin state. For the spin $5 / 2$, the calculated $\theta$ is however always outside the $8-12^{\circ}$ region, leading to $\Delta E_{\mathrm{QS}}$ values in restricted ranges of 1.4-1.6 $\mathrm{mm} \cdot \mathrm{s}^{-1}$ for $\mathrm{FeN}_{4} \mathrm{C}_{10}$ clusters and 0.6-1.1 $\mathrm{mm} \cdot \mathrm{s}^{-1}$ for $\mathrm{FeN}_{4} \mathrm{C}_{12}$ clusters (Table 2). As we will see below, experimentally and for the ferric species, only the high-spin is unambiguously identified in $\mathrm{Fe}_{0.5}-\mathrm{NC}$, which eases the assignments.

\subsection{Experimental results}

The experimental Mössbauer spectrum of $\mathrm{Fe}_{0.5}-\mathrm{NC}$ recorded at $80 \mathrm{~K}$, in the absence of external magnetic field, is shown in Figure 4a. The fitting was performed using two broad doublets with a Gaussian distribution of quadrupole splitting, and with an additional relaxed 
component to take into account the large background signal. We initially suspected this broad signal to come from Fe oxide nanoparticles, but it was almost unmodified at low and high temperature (5 K and $296 \mathrm{~K}$, Figure S3). This excludes its assignment as Fe oxide clusters, since such phases would show a sextet signal at $5 \mathrm{~K}$ and a doublet signal at $296 \mathrm{~K}$. While the fitting shown in Figure 4a could also have been performed without Gaussian distribution of the quadrupole splitting, this would have led to unusually high linewidths for D1 and D2, typically 0.7 and $1.3 \mathrm{~mm} \cdot \mathrm{s}^{-1}$, respectively, as reported by us for a similar catalyst, see Figure 1 and Table S1 in Ref. 10. We believe these high linewidths previously reported with such a simpler fitting approach arise from the electronic, chemical and structural disorder existing in pyrolyzed Fe-N-C catalysts. Here, we therefore opted for a fitting with a Gaussian distribution of quadrupole splittings, in order to more accurately compare all quadrupole splitting values experimentally observed to those calculated for a broad family of active sites. The results of the fitting are shown in Table S8. The two quadrupole doublet distributions have similar isomer shifts of 0.46 and $0.49 \mathrm{~mm} \cdot \mathrm{s}^{-1}$, but are distinguished by their average quadrupole splittings, centered at 0.94 and $2.25 \mathrm{~mm} \cdot \mathrm{s}^{-1}$ for D1 and D2, respectively. Such values are typically observed in Fe-N-C catalysts. ${ }^{5-10,14-15,24}$ While these two doublets in pyrolyzed Fe$\mathrm{N}-\mathrm{C}$ materials were clearly correlated to $\mathrm{FeN}_{4}$-type moieties with the help of X-ray absorption spectroscopy and HR-TEM ${ }^{10,14-15,22-23,62}$ their experimental values of isomer shift and quadrupole splittings do not allow an unambiguous assignment to a specific oxidation state or spin state of iron in such moieties. The isomer shift is in the range expected for both $\mathrm{Fe}(\mathrm{II})$ moieties in low and medium spin state, and for $\mathrm{Fe}(\mathrm{III})$ moieties in any spin state. $\Delta E_{\mathrm{QS}}$ values in the range 0.8 to $1.5 \mathrm{~mm} \cdot \mathrm{s}^{-1}$ can be assigned either to $\mathrm{Fe}$ (II) $\mathrm{S}=0$ or $\mathrm{Fe}$ (III) $\mathrm{S}=5 / 2$ species (Figure 2.32 in $\operatorname{Ref}^{24}$ ). From this result, only Fe in high oxidation states above + III ( $\delta$ value $\leq$ $\left.0.3 \mathrm{~mm} \mathrm{~s}^{-1}\right)$ and Fe(II) in high spin state $(\mathrm{S}=2)$ can be excluded $\left(\delta>0.9 \mathrm{~mm} \mathrm{~s}^{-1}\right){ }^{21}$ The exclusion of $\mathrm{Fe}$ in +IV or higher oxidation state is also and more clearly supported by XPS, showing only $\mathrm{Fe}$ in $+\mathrm{II}$ or $+\mathrm{III}$ oxidation states for atomically-dispersed $\mathrm{Fe}-\mathrm{N}-\mathrm{C}$ catalysts. 

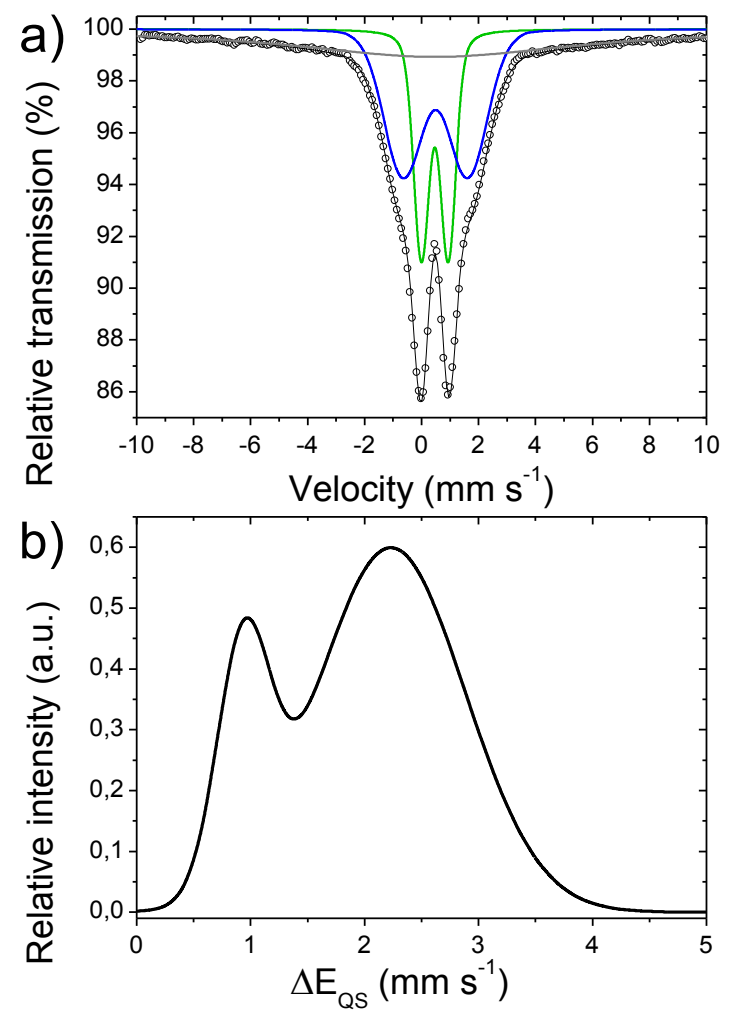

Figure 4. ${ }^{57} \mathrm{Fe}$ Mössbauer spectrum at $80 \mathrm{~K}$ of $\mathrm{Fe}_{0.5}$ - $\mathrm{NC}$ measured in air in the absence of external magnetic field (Fig. 4a) and corresponding distribution of the quadrupole splitting (Fig. 4b). The doublets D1 and D2 (and their fitted distribution of $\Delta E_{\mathrm{QS}}$ values) are highlighted in green and blue, respectively.

In order to gain more insights on the spin state of moieties present in this catalyst, we first characterized $\mathrm{Fe}_{0.5}-\mathrm{NC}$ with EPR. Figure $\mathrm{S} 4$ shows the X-band EPR spectra for $\mathrm{Fe}_{0.5}-\mathrm{NC}$ and for the corresponding blank measurement (no catalyst in the tube). The signal at circa $160 \mathrm{mT}$ ( $g=4.28$ ) in Figure $\mathrm{S} 4 \mathrm{~b}$ can be unambiguously assigned to a rhombic $\mathrm{S}=5 / 2 \mathrm{Fe}(\mathrm{III})$ center. $^{63}$ The six lines at 300-370 $\mathrm{mT}$ are assigned to Mn impurities. No specific signal is observed for $\mathrm{Fe}(\mathrm{III})$ in other spin states, nor for $\mathrm{Fe}(\mathrm{II})$, which is a non-Kramer ion and is normally EPRsilent. This information facilitates the further analysis of the Mössbauer spectrum, since we now can assume that $\mathrm{FeN}_{4}$ moieties are mainly present either as $\mathrm{Fe}$ (III) $\mathrm{S}=5 / 2$ high spin state, or as $\mathrm{Fe}(\mathrm{II})$ in the $\mathrm{S}=0$ or $\mathrm{S}=1$ spin states. The $\mathrm{Fe}(\mathrm{II}) \mathrm{S}=2$ state can be excluded, based on the above mentioned high isomer shift value expected for such a configuration, not experimentally observed.

The average molecular susceptibility of $\mathrm{Fe}_{0.5}-\mathrm{NC}$ was then measured by SQUID as a function of temperature (Figure S5a). The data could be fitted with a Curie-Weiss law, Eq. 2 (Figure S5b) with slightly negative $\theta_{\mathrm{C}}$-value, corresponding to a weak antiferromagnetic coupling. 
The constant $\mathrm{C}$ can be then further analyzed assuming two different types of Fe centers in $\mathrm{Fe}_{0.5}-\mathrm{NC}$, knowing that their $\chi$ values are additive, using Eqs. 2 and 3:

$\chi_{\mathrm{m}}^{-1}=\left(\mathrm{T}-\theta_{\mathrm{C}}\right) / \mathrm{C}$

$\mu_{\text {eff }}^{2}=\mu_{\mathrm{B}}^{2}\left[\mathrm{a}_{1}\left(\mathrm{~s}_{1}^{2}+\mathrm{s}_{1}\right)+\mathrm{a}_{2}\left(\mathrm{~s}_{2}^{2}+\mathrm{s}_{2}\right)\right]$

where $a_{1}$ and $a_{2}$ are the molar fractions of $\mathrm{Fe}$ in the two types of sites, $s_{1}$ and $s_{2}$ are their corresponding spin states, $\mu_{\text {eff }}$ the average effective magnetic moment of iron atoms. With the fitted value of constant $C$ (2.566), the second term in Eq. 3 should be equal to 20.4 to match the experimental fitting. Assuming $\mathrm{Fe}_{0.5}-\mathrm{NC}$ contains a mix of iron centers with only $\mathrm{Fe}(\mathrm{II})$ in $\mathrm{S}=0$ state and $\mathrm{Fe}(\mathrm{III})$ in $\mathrm{S}=5 / 2$ state, the value of 20.4 is obtained with a relative fraction of $58 \%$ of high spin Fe(III). Assuming a mix of only $\mathrm{Fe}$ (II) in $\mathrm{S}=1$ state and Fe(III) $\mathrm{S}=5 / 2$ state, a match is obtained with a relative fraction of $46 \%$ high spin $\mathrm{Fe}(\mathrm{III})$. These results are consistent with the relative fraction from Mössbauer (Table S8), if one assumes that both D1 and the broad relaxation background are in high-spin ferric state, and D2 a ferrous center in zero-spin state.

To obtain further information on the spin state of the doublets D1 and D2, Mössbauer spectroscopy measurements were then performed in the presence of an external magnetic field. The behavior of the Mössbauer nuclei under an applied field is strongly dependent on their spin state. In the case of diamagnetic nuclei, i.e. with $S=0$, the measured field is equal to the applied external field, whereas in the case of paramagnetic ions the effective measured field is equal to the applied field plus the hyperfine field which is created by the magnetization. ${ }^{59}$ In the case of high spin $\mathrm{Fe}(\mathrm{III})$, due to the large magnetic moment corresponding to the $S=5 / 2$ state, very high effective fields exceeding $50 \mathrm{~T}$ can be obtained. Figure 5 shows the spectra of $\mathrm{Fe}_{0.5}-\mathrm{NC}$ measured with an external magnetic field of $7 \mathrm{~T}$ applied either parallel or perpendicular to the $\gamma$-rays as well as the corresponding distribution of hyperfine magnetic fields obtained by the common fit of the two spectra. Interestingly, two maxima centered at about $7 \mathrm{~T}$ and $45 \mathrm{~T}$ are observed in the distribution. The second value unambiguously underlines the presence of high spin Fe(III), confirming both SQUID and EPR results. While hyperfine fields exceeding $50 \mathrm{~T}$ are commonly measured for slowly relaxing $\mathrm{Fe}(\mathrm{III})$ ions, the external magnetic field usually couples antiferromagnetically to the paramagnetic relaxation field, thus decreasing the observed value of the hyperfine field. This 
explains the strong signal observed at $45 \mathrm{~T}$. On the other hand, the component centered around $7 \mathrm{~T}$ could derive from the presence of diamagnetic low spin $\mathrm{Fe}(\mathrm{II})$. In fact, a value of the hyperfine field equaling the value of the applied field is a rather clear sign of the presence of a diamagnetic ion, since no additional fields caused by the magnetization of unpaired electrons are experienced by the Fe ions.

It must also be said that the fit shown in Figure 5, obtained using a distribution of magnetic sextets, does not describe precisely the structure of the two spectra acquired at low values of external magnetic fields (not shown). This is not unexpected since, for low values of hyperfine field and relatively high values of quadrupole splitting, the quadrupole interaction cannot be treated anymore as a perturbation of the Zeeman effect caused by the magnetic field, and the full Hamiltonian including both interactions must be solved. ${ }^{64}$ In this case, the experimental spectrum strongly depends upon the mutual orientation of the $\mathrm{V}_{\mathrm{zz}}$ component of the EFG and of the magnetic field, which is virtually impossible to determine in such broadened multicomponent spectra. However, in the absence of a local magnetic moment on the absorbing ions, which are randomly oriented in the sample, one can assume a random orientation of the magnetic field with respect to the EFG. ${ }^{64}$ The theoretical spectrum of such a system, calculated assuming a hyperfine field of $7 \mathrm{~T}$ and a EFG of $2.3 \mathrm{~mm} \cdot \mathrm{s}^{-1}$ $\left(\mathrm{V}_{\mathrm{zz}}=2.3 \mathrm{~mm} \cdot \mathrm{s}^{-1}, \eta=0\right.$, simulating doublet $\mathrm{D} 2$ in the spectrum without applied external magnetic field), is shown in Figure S6. As it can be clearly seen, the features of the central part of the spectrum (central peak, slight asymmetry and intensity of the two central lines) correspond rather well to the calculated spectrum.

This detailed analysis of the Mössbauer spectrum under external magnetic field supports i) the presence of a substantial fraction of diamagnetic Fe(II) ions in the sample, with a EFG close to $2.3 \mathrm{~mm} \cdot \mathrm{s}^{-1}$ and ii) its assignment to the experimentally observed D2 signal in the spectrum without applied external field. This in turn implies that D1 can, on the other hand, be assigned in majority to $\mathrm{Fe}(\mathrm{III}) \mathrm{N}_{4}$ sites with high spin. In spite of these main attributions, the presence of other Fe sites in minor content cannot be excluded. If no additional signal typical of other $\mathrm{Fe}(\mathrm{III})$ sites in $\mathrm{S}=3 / 2$ or $1 / 2$ states is detected by EPR, a minor amount of $\mathrm{Fe}$ (II) $\mathrm{S}=1$ species cannot be experimentally excluded. 

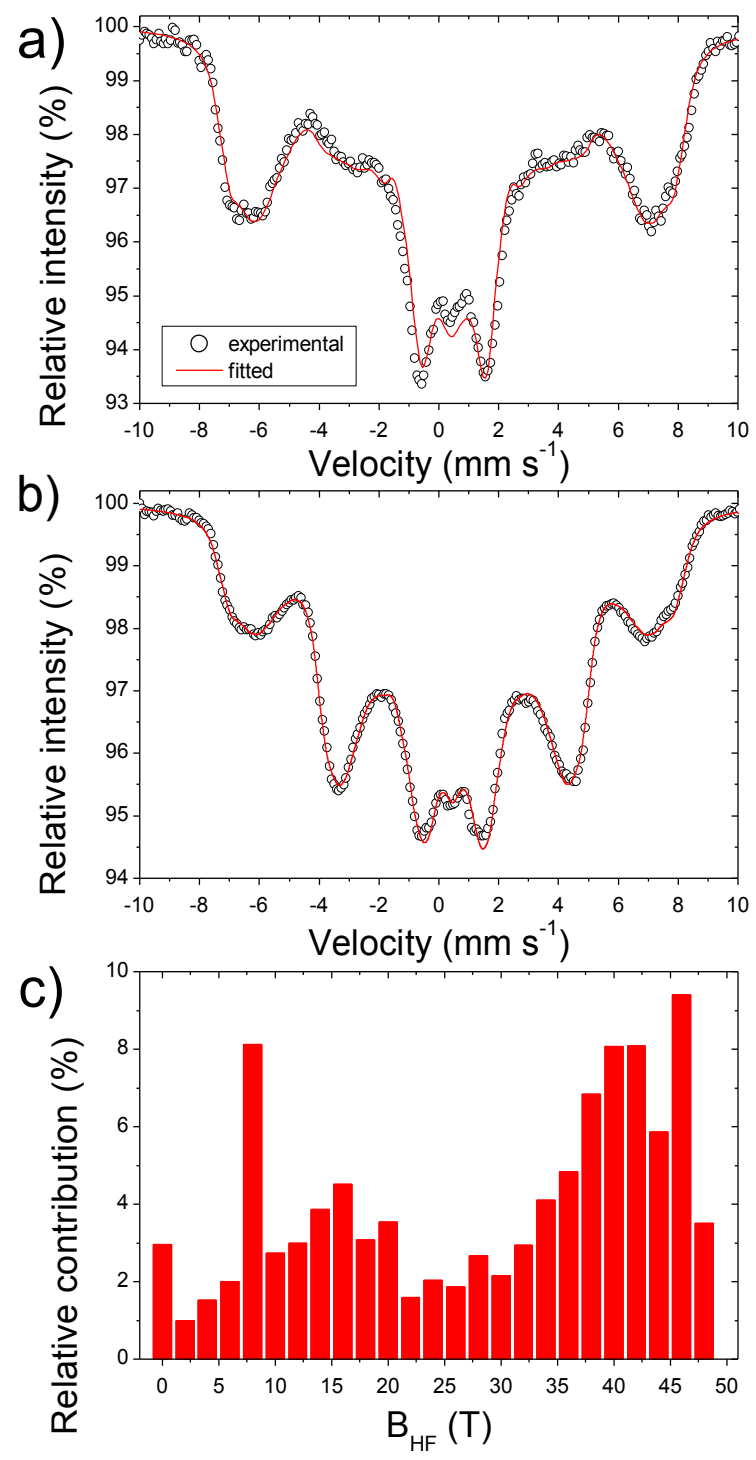

Figure 5. ${ }^{57} \mathrm{Fe}$ Mössbauer spectra of $\mathrm{Fe}_{0.5}-\mathrm{NC}$ measured at $4.5 \mathrm{~K}$ with an external magnetic field of $7 \mathrm{~T}$ applied in the direction a) parallel or b) perpendicular to the $\gamma$-rays, and c) the corresponding distribution of hyperfine fields from the common fit of the two spectra using a distribution of magnetic sextets (with a same isomer shift). The fits in a) and b) were obtained with the single distribution of hyperfine magnetic fields shown in c) (the common fitted isomer shift was $\delta=0.47 \mathrm{~mm} \cdot \mathrm{s}^{-1}$ ), and with line intensity ratios of 3:2x:1:1:2x:3 with fitted $\mathrm{x}$ values of 0.29 and 1.90 for the parallel and perpendicular field spectrum, respectively. 


\section{Discussion}

To compare experimental and theoretical insights gained in this study, we focus here on the DFT results obtained on the 1a, 1d, 1e and 1f clusters with the PBE/DZVP2 and PBE/Wachters methods. The main conclusions would not be changed if all DFT results are considered, including those obtained with the periodic approaches. Figure 6 shows the calculated $\Delta E_{\mathrm{QS}}$ values for the above mentioned combinations of clusters and DFT methods, with the $\mathrm{FeN}_{4}$ clusters in three possible combinations of oxidation and spin state, namely $\mathrm{Fe}(\mathrm{II}) \mathrm{S}=0, \quad \mathrm{Fe}$ (II) $\mathrm{S}=1$ and $\mathrm{Fe}(\mathrm{III}) \mathrm{S}=5 / 2$. These combinations correspond to those identified as major (Fe(II) $\mathrm{S}=0$, and $\mathrm{Fe}(\mathrm{III}) \mathrm{S}=5 / 2$ ) or possible minor species (Fe(II) $\mathrm{S}=$ 1) for the present Fe-N-C catalyst by experimental Mössbauer spectroscopy, SQUID and EPR.

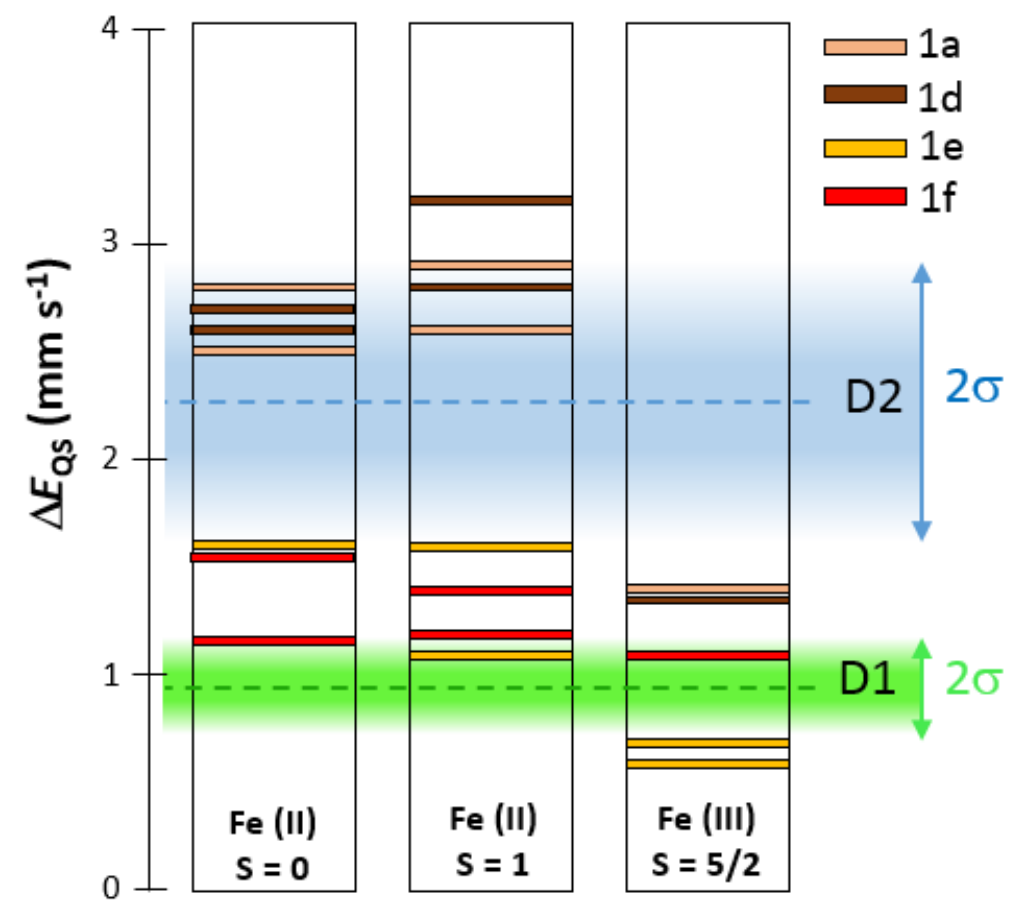

Figure 6. Comparison between DFT-calculated and measured quadrupole splittings for $\mathrm{FeN}_{\mathrm{x}}$ sites. $\Delta E_{\mathrm{QS}}$ values calculated with the PBE/DZVP2 and PBE/Wachters methods for the 1a, 1d, 1e and $\mathbf{1 f}$ clusters are presented. The green and blue areas correspond to the experimentally measured distribution of the doublets D1 and D2, and the dashed lines correspond to the center of the fitted Gaussian distribution for each of them $\left(0.94\right.$ and $2.25 \mathrm{~mm} \cdot \mathrm{s}^{-1}$ respectively). The colored D1 and D2 areas expand $\sigma$ (where $\sigma$ is their standard deviation, see Table S8) above and below the mean $\Delta E_{\mathrm{QS}}$ value fitted for D1 and D2, thereby representing $68 \%$ of the entire D1 and D2 signal fitted with the broad doublet Gaussian distribution in figure 4. 
From the comparison of experimental and calculated $\Delta E_{\mathrm{QS}}$ values only, Figure 6 shows that the experimental doublet D1 can only be assigned to $\mathrm{FeN}_{4} \mathrm{C}_{12}$ sites (1e and $\mathbf{1 f}$ clusters, represented by red and orange horizontal bars), but in any of the three above-mentioned oxidation/spin combinations. The center of the D1 distribution $\left(0.94 \mathrm{~mm} \cdot \mathrm{s}^{-1}\right.$, dashed green line) best corresponds however to $\mathrm{Fe}(\mathrm{III}) \mathrm{N}_{4} \mathrm{C}_{12}$ sites (1e and $\mathbf{1 f}$ in Figure 6) with high spin. When considering periodic DFT calculations, it should be noted that high spin $\mathrm{Fe}(\mathrm{III}) \mathrm{N}_{4} \mathrm{C}_{10}$ and $\mathrm{Fe}(\mathrm{III}) \mathrm{N}_{4} \mathrm{C}_{12}$ have identical $\Delta E_{\mathrm{QS}}$ values $\left(0.7 \mathrm{~mm} \mathrm{~s}^{-1}\right)$, that can be assigned to the lower end of the D1 signal. All this supports the main assignment of D1 to Fe(III) $S=5 / 2$ moieties earlier in this work, that we derived from the analysis of the experimental Mössbauer spectrum under external magnetic field. The $1 \mathbf{a}$ and $\mathbf{1 d}$ clusters $\left(\mathrm{FeN}_{4} \mathrm{C}_{10}\right.$ sites $)$ in $\mathrm{Fe}(\mathrm{III}) \mathrm{S}=$ $5 / 2$ state fall in-between the D1 and D2 regions shown in Figure 6 and that correspond to the mean $\Delta E_{\mathrm{QS}}$ value of $\mathrm{D} 1$ and $\mathrm{D} 2 \pm \sigma$, i.e. representing $68 \%$ of the total $\mathrm{D} 1$ and $\mathrm{D} 2$ signal as fitted in Figure 4a. Thus, they may contribute to the broadening of the D1 and D2 doublets in the region $1.2-1.4 \mathrm{~mm} \cdot \mathrm{s}^{-1}$. The overlap in this region is clearly seen in the complete $\Delta E_{\mathrm{QS}}$ distributions for D1 and D2 shown in Figure 4b. In contrast, D2 can mostly be assigned to $\mathrm{Fe}(\mathrm{II}) \mathrm{N}_{4} \mathrm{C}_{10}$ sites, in either $\mathrm{S}=0$ or $\mathrm{S}=1$ spin state. The center of the D2 distribution $(2.25$ $\mathrm{mm} \cdot \mathrm{s}^{-1}$, dashed blue line) slightly better corresponds however to $\mathrm{Fe}(\mathrm{II}) \mathrm{N}_{4} \mathrm{C}_{10}$ sites with low spin. This also supports the main assignment of D2 to Fe(II) $S=0$ moieties earlier in this work, that was derived from the analysis of the experimental Mössbauer spectrum under external magnetic field. We however do not exclude the presence of a minor content of $\mathrm{Fe}$ (II) $\mathrm{S}=1$ sites, and such sites would, according to our DFT results, either clearly contribute to the D2 experimental signal $\left(\mathrm{FeN}_{4} \mathrm{C}_{10}\right.$ sites), or to $\mathrm{D} 1$ and the intermediate region overlapping D1 and $\mathrm{D} 2\left(\mathrm{FeN}_{4} \mathrm{C}_{12}\right.$ sites), see Figure 6, central column.

It can thus be concluded from this comparison between calculated and experimental $\Delta E_{\mathrm{QS}}$ values, and from EPR, SQUID and from the analysis of the Mössbauer spectrum under external magnetic field, that the D1 and D2 experimental signals can mostly be assigned to $\mathrm{Fe}(\mathrm{III}) \mathrm{N}_{4} \mathrm{C}_{12}$ sites with high spin and $\mathrm{Fe}(\mathrm{II}) \mathrm{N}_{4} \mathrm{C}_{10}$ sites with low spin, respectively. Because of the high relative content of $\mathrm{Fe}(\mathrm{II}) \mathrm{S}=0$ and $\mathrm{Fe}$ (III) $\mathrm{S}=5 / 2$ species experimentally identified, and in view of Figure 6, it is difficult to propose other main assignments. For example, while from Figure 6 alone, D1 could be assigned mostly to $\mathrm{Fe}(\mathrm{II}) \mathrm{N}_{4} \mathrm{C}_{12}$ sites with $\mathrm{S}=0$ and D2 mostly to $\mathrm{Fe}(\mathrm{II}) \mathrm{N}_{4} \mathrm{C}_{10}$ sites with $\mathrm{S}=1$, there is then no main assignment for an $\mathrm{Fe}$ (III) $\mathrm{S}=5 / 2$ species. However, the latter is identified experimentally by Mössbauer under external 
magnetic field and EPR, while SQUID also supports the presence of a high relative fraction of high spin ferric species.

These main assignments of D1 and D2 are important novel insights, and in strong contrast to previous empirical assignments of D1 and D2. ${ }^{5-10,24}$ It is here recalled that the ambiguity and lack of evidence of those previous assignments was generally recognized by those involved in the field. It also implies that the D1 signal must be located on the top surface of the carbon matrix, thereby accessible to gas-phase, leading to ferric species as soon as the sample is exposed to air. The D2 signal, assigned to ferrous species, may then be explained by the inaccessibility of such sites to the ambient air, i.e. such sites might be viewed as being buried underneath the surface, or on the surface but with weaker oxygen binding. These assignments are well supported by recent results obtained by Kneebone et al on the effect of NO adsorption on the Mössbauer spectrum of another Fe-N-C catalyst. ${ }^{65}$ In that study, the doublet with lowest $\Delta E_{\mathrm{QS}}$ in the pristine catalyst $\left(\Delta E_{\mathrm{QS}}=0.83 \mathrm{~mm} \cdot \mathrm{s}^{-1}, \delta=0.30 \mathrm{~mm} \cdot \mathrm{s}^{-1}\right.$ and labelled D4 in that work but corresponding to D1 label in the present work) was found to explain most of the spectral changes observed upon the electrochemical reduction of the catalyst, and then upon NO adsorption on the surface ferrous sites. That study and the present one agree then in identifying the D1 signal in the Mössbauer spectrum of Fe-N-C catalysts exposed to air as a ferric, surface-located species, which can be reduced to ferrous form when the applied electrochemical potential is lower than that of the $\mathrm{Fe}(\mathrm{III}) / \mathrm{Fe}(\mathrm{II})$ redox couple of such $\mathrm{FeN} \mathrm{N}_{4}$ sites. The ferric state of D1 sites when exposed to air and their ability to be reduced to ferrous state when the electrochemical potential is lowered in the ORR range indicates that such sites are likely the ORR active sites in Fe-N-C catalysts. Again, this is supported by previous positive correlations between the absolute or relative amount of D1 signal and the ORR activity for different series of Fe-N-C catalysts obtained by varying one single experimental synthesis parameter (e.g. Fe content, pyrolysis duration, pyrolysis temperature, etc). ${ }^{9,} 66$ The assignment of the D1 signal to high-spin $\mathrm{Fe}(\mathrm{III}) \mathrm{N}_{4} \mathrm{C}_{12}$ moieties is also in agreement with similar $\Delta E_{\mathrm{QS}}$ and $\delta$ values as $\mathrm{D} 1$ observed for a monolayer coverage of Fe-phthalocyanine $(\mathrm{FePc})$ on Vulcan carbon black $\left(\Delta E_{\mathrm{QS}}=0.96\right.$ and $\delta=0.40$, Table 1 in Ref ${ }^{67}$. In that work, the author assigned this new doublet (different from that of crystalline FePc) to the binding of $\mathrm{FePc}$ to the carbon substrate through an oxygen atom of a carbon functional group. But it appears simpler now to assign this "new" spectral feature of FePc to the fact that all Fe centers in this monolayer coverage configuration are exposed to $\mathrm{O}_{2}$ from ambient air, which is 
impossible in crystalline FePc. Following our thinking, the D2 signal in pyrolyzed Fe-N-C catalysts has similar Mössbauer parameters to bulk crystalline $\operatorname{FePc}\left(\Delta E_{\mathrm{QS}}=2.61\right.$ and $\delta=$ 0.39 , Table 1 in $^{67}$. This supports the idea that D2 may be assigned either i) to similar $\mathrm{FeN}_{4}$ sites as D1, but located in the bulk of the carbon matrix and thus inaccessible to the gas-phase (and the liquid electrolyte) impeding their oxidation by $\mathrm{O}_{2}$, or ii) to $\mathrm{FeN}_{4}$ surface sites with a $\mathrm{Fe}(\mathrm{III}) / \mathrm{Fe}$ (II) redox potential sufficiently high that their stable form is $\mathrm{Fe}$ (II), even when exposed to air in open circuit conditions. Last, the assignment of D1 to, mostly, a ferric high spin moiety is also in line with a recent study from Kramm's group, involving experimental ${ }^{57} \mathrm{Fe}$ Mössbauer spectroscopy at $5 \mathrm{~K}$ with and without perpendicular applied magnetic field, as well as EPR and nuclear inelastic spectroscopy. ${ }^{68}$ The major presence of a high-spin ferric moiety ( $28 \%$ of the absorption signal) was evidenced, and correlated with a doublet with $\Delta \mathrm{E}_{\mathrm{QS}}$ of $1.0 \mathrm{~mm} \mathrm{~s}^{-1}$ (Table 2 in that work), corresponding to our D1 component, while a doublet with even smaller $\Delta \mathrm{E}_{\mathrm{QS}}$ value $\left(0.72 \mathrm{~mm} \mathrm{~s}^{-1}\right)$ was assigned to a ferrous low-spin moiety, but representing only $12 \%$ of the absorption signal. This is coherent with our Figure 6 , showing that porphyrinic moieties (1e-1f clusters) can result in low $\Delta \mathrm{E}_{\mathrm{QS}}$ values of ca 1 $\mathrm{mm} \mathrm{s}^{-1}$ either in ferric high-spin or ferrous low spin states.

This work thus identifies D1 as surface sites which, combined with a precise measurement of the Fe bulk content and applying correct Lamb-Mössbauer factors for D1 and D2 and any other Fe species present in a given Fe-N-C catalyst, ${ }^{13}$ can directly yield the absolute number of $\mathrm{Fe}(\mathrm{D} 1)$ surface sites. Due to the $\Delta E_{\mathrm{QS}}$ distribution within the broad D1 signal, it may however be that not all D1 sites are similarly active toward ORR, and some may be unstable, at least in acidic medium. Following the fate of D1 signal during electrochemical operation will be necessary to more closely connect it to the ORR activity after short break-in of the Fe$\mathrm{N}-\mathrm{C}$ electrode in electrolyte, and then also after longer operation in liquid-electrolyte or in fuel cell.

The experimental and theoretical methods reported here to study in detail $\mathrm{FeN}_{\mathrm{x}}$ sites embedded in graphene for fuel cell application are also of interest for other electrochemical applications such as $\mathrm{CO}_{2}$ electro-reduction ${ }^{69}$, nitrite and nitric oxide electroreduction ${ }^{70}$ but also for the broader field of heterogeneous catalysis, where similar sites have been reported to catalyze a range of interesting reactions for high added-value products. ${ }^{71-73}$ 


\section{Conclusions}

- Calculated quadrupole splitting values for ferrous moieties ( $S=0$ or $S=1$ spin state) clearly differentiate $\mathrm{FeN}_{4} \mathrm{C}_{12}\left(<1.8 \mathrm{~mm} \cdot \mathrm{s}^{-1}\right)$ from $\mathrm{FeN}_{4} \mathrm{C}_{10}$ moieties $\left(>2.4 \mathrm{~mm} \cdot \mathrm{s}^{-1}\right)$

- No significant effect of cluster size on calculated quadrupole splitting value for ferrous $\mathrm{FeN}_{4} \mathrm{C}_{\mathrm{y}}$ moieties $(\mathrm{S}=0$ or $\mathrm{S}=1$ spin state)

- Calculated quadrupole splitting value for ferric $\mathrm{FeN}_{4} \mathrm{C}_{\mathrm{y}}$ moieties in $\mathrm{S}=3 / 2$ spin state mainly depends on $\mathrm{OH}$-orientation, less on model structure

- Calculated quadrupole splitting values for ferric moieties in $S=5 / 2$ spin state weakly differentiate $\mathrm{FeN}_{4} \mathrm{C}_{12}\left(<1.2 \mathrm{~mm} \cdot \mathrm{s}^{-1}\right)$ from $\mathrm{FeN}_{4} \mathrm{C}_{10}$ moieties $\left(>1.3 \mathrm{~mm} \cdot \mathrm{s}^{-1}\right)$

- Experimental identification of the presence of $\mathrm{Fe}$ (II) $\mathrm{S}=0$ and $\mathrm{Fe}$ (III) $\mathrm{S}=5 / 2$ centers in Fe-N-C catalyst with Mössbauer spectroscopy under strong external magnetic field, EPR and SQUID

- Assignment of experimental doublet 1 (with mean quadrupole splitting value at 0.94 $\mathrm{mm} \cdot \mathrm{s}^{-1}$ ) to mostly $\mathrm{Fe}(\mathrm{III}) \mathrm{N}_{4} \mathrm{C}_{12}$ sites with $\mathrm{S}=5 / 2$ and of the doublet 2 (with mean quadrupole splitting value at $2.25 \mathrm{~mm} \cdot \mathrm{s}^{-1}$ ) to mostly $\mathrm{Fe}(\mathrm{II}) \mathrm{N}_{4} \mathrm{C}_{10}$ sites with $\mathrm{S}=0$, through combined experimental and theoretical results

- Identification of experimental doublet 1 as $\mathrm{FeN}_{\mathrm{x}}$ sites located on top surface and as a major contributor of ORR activity

\section{Supporting information}

Examples of structures optimized with PBE/DZVP2 level of theory (Figure S1), table of population of orbitals for $1 \mathrm{a}$ and $2 \mathrm{a}$ ferrous models in $\mathrm{S}=1$ spin state (Table $\mathrm{S} 1$ ) and their structural parameters for $S=0$ and $S=1$ (Table $S 2$ ), table of structural parameters for ferrous 1e and 2e models for $S=0$ and $S=1$ optimized with different DFT methods (Table S3), effect of cluster size on quadrupole splitting, relative energies and structural parameters for ferrous models (Table S4-S5), scheme of possible $\mathrm{OH}$ orientation on a ferric $\mathrm{FeN}_{4} \mathrm{C}_{\mathrm{y}}$ model site (Figure S2), structural parameters of ferric $\mathrm{FeN}_{4} \mathrm{C}_{10}$ and $\mathrm{FeN}_{4} \mathrm{C}_{12}$ clusters for different spin states and with different DFT methods (Table S6-S7), results of fitting of experimental Mössbauer spectra (Table S8), effect of temperature on Mössbauer spectra (Figure S3), Xband EPR spectra (Figure S4), SQUID measurement (Figure S5), experimental Mössbauer spectra with external magnetic field applied parallel or perpendicular to $\gamma$-rays and theoretical simulation for randomly oriented Fe(II) ions creating a quadrupole splitting of $2.3 \mathrm{~mm} \mathrm{~s}$ (Figure S6),

\section{Acknowledgements}


This work was partially funded by the French National Research Agency (Labex programme CheMISyst, grant agreement ANR-10-LABX-05-01) and the FCH Joint Undertaking (CRESCENDO Project, Grant Agreement n779366). T.M. acknowledges GENCI-CCRT center (project No. A0050807369) for a generous allocation of high performance computing resources. Corine Reibel (ICGM UMR 5253 CNRS) is acknowledged for her help with SQUID measurements. I.M. thankfully acknowledges the computational resources of the National Energy Research Scientific Computing Center (NERSC), a U.S. Department of Energy Office of Science User Facility operated under Contract No. DE-AC02-05CH11231 and UNM Center for Advanced Research Computing (CARC). This paper has been designated LA-UR-19-27987. 


\section{References}

1. de Souza Júnior, P. A. Extraterrestrial and Terrestrial Outdoor Applications of Mössbauer Spectroscopy. Johannes Gutenberg-Universität Mainz, 2004.

2. Mössbauer Spectroscopy: Applications in Chemistry, Biology and Nanotechnology. John Wiley \& sons Inc. : 2013.

3. Niemantsverdriet, J. W.; Van der Kraan, A. M.; Van Dijk, W. L.; Van der Baan, H. S., Behavior of Metallic Iron Catalysts During Fischer-Tropsch Synthesis Studied with Moessbauer Spectroscopy, X-ray Diffraction, Carbon Content Determination, and Reaction Kinetic Measurements. J. Phys. Chem. 1980, 84, 3363-3370.

4. Rao, K. R. P. M.; Huggins, F. E.; Mahajan, V.; Huffman, G. P.; Rao, V. U. S.; Bhatt, B. L.; Bukur, D. B.; Davis, B. H.; O'Brien, R. J., Mössbauer Spectroscopy Study of Ironbased Catalysts Used in Fischer-Tropsch Synthesis. Topics in Catalysis 1995, 2, 71-78.

5. Schulenburg, H.; Stankov, S.; Schünemann, V.; Radnik, J.; Dorbandt, I.; Fiechter, S.; Bogdanoff, P.; Tributsch, H., Catalysts for the Oxygen Reduction from Heat-Treated Iron(III) Tetramethoxyphenylporphyrin Chloride: Structure and Stability of Active Sites. J. Phys. Chem. B 2003, 107, 9034-9041.

6. Kramm, U. I.; Herranz, J.; Larouche, N.; Arruda, T. M.; Lefevre, M.; Jaouen, F.; Bogdanoff, P.; Fiechter, S.; Abs-Wurmbach, I.; Mukerjee, S.; Dodelet, J.-P., Structure of the Catalytic Sites in Fe/N/C-catalysts For O2-reduction in PEM Fuel Cells. Phys. Chem. Chem. Phys. 2012, 14, 11673-11688.

7. Kramm, U. I.; Abs-Wurmbach, I.; Herrmann-Geppert, I.; Radnik, J.; Fiechter, S.; Bogdanoff, P., Influence of the Electron-Density of FeN4-Centers Towards the Catalytic Activity of Pyrolyzed FeTMPPCl-Based ORR-Electrocatalysts. J. Electrochem. Soc. 2011, 158, B69-B78.

8. Bouwkamp-Wijnoltz, A. L.; Visscher, W.; van Veen, J. A. R.; Boellaard, E.; van der Kraan, A. M.; Tang, S. C., On Active-Site Heterogeneity in Pyrolyzed Carbon-Supported Iron Porphyrin Catalysts for the Electrochemical Reduction of Oxygen: An In Situ Mössbauer Study. J. Phys. Chem. B 2002, 106, 12993-13001.

9. Koslowski, U. I.; Abs-Wurmbach, I.; Fiechter, S.; Bogdanoff, P., Nature of the Catalytic Centers of Porphyrin-Based Electrocatalysts for the ORR: A Correlation of Kinetic Current Density with the Site Density of Fe-N4 Centers. J. Phys. Chem. C 2008, 112, 1535615366 .

10. Zitolo, A.; Goellner, V.; Armel, V.; Sougrati, M.-T.; Mineva, T.; Stievano, L.; Fonda, E.; Jaouen, F., Identification of Catalytic Sites for Oxygen Reduction in Iron- and Nitrogendoped Graphene Materials. Nat. Mater. 2015, 14, 937.

11. Sahraie, N. R.; Kramm, U. I.; Steinberg, J.; Zhang, Y.; Thomas, A.; Reier, T.; Paraknowitsch, J.-P.; Strasser, P., Quantifying the Density and Utilization of Active Sites in Non-precious Metal Oxygen Electroreduction Catalysts. Nat. Commun. 2015, 6, 8618.

12. Goellner, V.; Baldizzone, C.; Schuppert, A.; Sougrati, M. T.; Mayrhofer, K.; Jaouen, F., Degradation of Fe/N/C Catalysts Upon High Polarization in Acid Medium. Phys. Chem. Chem. Phys. 2014, 16, 18454-18462. 
13. Sougrati, M. T.; Goellner, V.; Schuppert, A. K.; Stievano, L.; Jaouen, F., Probing Active Sites in Iron-based Catalysts for Oxygen Electro-reduction: A Temperature-dependent 57Fe Mössbauer Spectroscopy Study. Catal. Today 2016, 262, 110-120.

14. Jia, Q.; Ramaswamy, N.; Tylus, U.; Strickland, K.; Li, J.; Serov, A.; Artyushkova, K.; Atanassov, P.; Anibal, J.; Gumeci, C.; Barton, S. C.; Sougrati, M.-T.; Jaouen, F.; Halevi, B.; Mukerjee, S., Spectroscopic Insights Into the Nature of Active Sites in Iron-Nitrogen-Carbon Electrocatalysts for Oxygen Reduction in Acid. Nano Energy 2016, 29, 65-82.

15. Serov, A.; Artyushkova, K.; Niangar, E.; Wang, C.; Dale, N.; Jaouen, F.; Sougrati, M.T.; Jia, Q.; Mukerjee, S.; Atanassov, P., Nano-structured Non-platinum Catalysts for Automotive Fuel Cell Application. Nano Energy 2015, 16, 293-300.

16. Strickland, K.; Miner, E.; Jia, Q.; Tylus, U.; Ramaswamy, N.; Liang, W.; Sougrati, M.-T.; Jaouen, F.; Mukerjee, S., Highly Active Oxygen Reduction Non-platinum Group Metal Electrocatalyst Without Direct Metal-Nitrogen Coordination. Nat. Commun. 2015, 6, 7343.

17. Kramm, U. I.; Ni, L.; Wagner, S., 57Fe Mössbauer Spectroscopy Characterization of Electrocatalysts. Advanced Materials 2019, 0, 1805623.

18. Goldanskii, V. I.; Herber, R. H., Chemical Applications of Mössbauer Spectroscopy. Academic Press: New York and London, 1968.

19. Maddock, A., Mössbauer Spectroscopy Principles and Applications. Horwood Publishing: Chichester, 1997.

20. Martinho, M.; Münck, E., 57Fe Mössbauer Spectroscopy in Chemistry and Biology. In Physical Inorganic Chemistry, John Wiley \& Sons, Inc.: 2010.

21. Stievano, L.; Wagner, F. E., Mössbauer Spectroscopy. Characterization of Solid Materials and Heterogeneous Catalysts 2012.

22. Li, J.; Jaouen, F., Structure and Activity of Metal-centered Coordination Sites in Pyrolyzed Metal-Nitrogen-Carbon Catalysts for the Electrochemical Reduction of O2. Current Opinion in Electrochemistry 2018, 9, 198-206.

23. Fei, H.; Dong, J.; Feng, Y.; Allen, C. S.; Wan, C.; Volosskiy, B.; Li, M.; Zhao, Z.; Wang, Y.; Sun, H.; An, P.; Chen, W.; Guo, Z.; Lee, C.; Chen, D.; Shakir, I.; Liu, M.; Hu, T.; Li, Y.; Kirkland, A. I.; Duan, X.; Huang, Y., General Synthesis and Definitive Structural Identification of MN4C4 Single-atom Catalysts with Tunable Electrocatalytic Activities. Nat. Catal. 2018, 1, 63-72.

24. Jaouen, F., Heat-Treated Transition Metal-NxCy Electrocatalysts for the O2 Reduction Reaction in Acid PEM Fuel Cells. In Non-Noble Metal Fuel Cell Catalysts, Chen, Z.; Dodelet, J.-P.; Zhang, J., Eds. Wiley VCH: Weinheim, Germany, 2014.

25. Fricke, B.; Waber, J. T., Calculation of Isomer Shift in Mössbauer Spectroscopy. Phys. Rev. B 1972, 5, 3445-3449.

26. Trautwein, A.; Harris, F. E.; Freeman, A. J.; Desclaux, J. P., Relativistic Electron Densities and Isomer Shifts of Fe57 in Iron-oxygen and Iron-fluorine Clusters and of Iron in Solid Noble Gases. Phys. Rev. B 1975, 11, 4102-4105.

27. Neese, F., Prediction and Interpretation of the 57Fe Isomer Shift in Mossbauer Spectra by Density Functional Theory. Inor. Chim. Acta 2002, 337, 181-192. 
28. Filatov, M., First Principles Calculation of Mössbauer Isomer Shift. Coord. Chem. Rev. 2009, 253, 594-605.

29. Nemykin, V. N.; Hadt, R. G., Influence of Hartree-Fock Exchange on the Calculated Mössbauer Isomer Shifts and Quadrupole Splittings in Ferrocene Derivatives Using Density Functional Theory. Inorg. Chem. 2006, 45, 8297-8307.

30. Bochevarov, A. D.; Friesner, R. A.; Lippard, S. J., Prediction of 57Fe Mössbauer Parameters by Density Functional Theory: A Benchmark Study. J. Chem. Theory Comput. 2010, 6, 3735-3749.

31. McWilliams, S. F.; Brennan-Wydra, E.; MacLeod, K. C.; Holland, P. L., Density Functional Calculations for Prediction of 57Fe Mössbauer Isomer Shifts and Quadrupole Splittings in $\beta^{-}$Diketiminate Complexes. ACS Omega 2017, 2, 2594-2606.

32. Zhang, Y.; Mao, J.; Oldfield, E., 57Fe Mössbauer Isomer Shifts of Heme Protein Model Systems: Electronic Structure Calculations. J. Am. Chem. Soc. 2002, 124, 7829-7839.

33. Pápai, M.; Vankó, G., On Predicting Mössbauer Parameters of Iron-Containing Molecules with Density-Functional Theory. J. Chem. Theory Comput. 2013, 9, 5004- 5020.

34. Sinnecker, S.; Slep, L. D.; Bill, E.; Neese, F., Performance of Nonrelativistic and Quasi-relativistic Hybrid DFT for the Prediction of Electric and Magnetic Hyperfine Parameters in 57Fe Mössbauer Spectra. Inorg. Chem. 2005, 44, 2245-2254.

35. Zhang, Y.; Oldfield, E., An Investigation of the Unusual 57Fe Mössbauer Quadrupole Splittings and Isomer Shifts in 2 and 3-Coordinate Fe(II) Complexes. J. Phys. Chem. B 2003, 107, 7180-7188.

36. Han, W.-G.; Noodleman, L., DFT Calculations of Comparative Energetics and ENDOR/Mössbauer Properties for Two Protonation States of the Iron Dimer Cluster of Ribonucleotide Reductase Intermediate X. Dalton Trans. 2009, 30, 6045-6057.

37. Han, W.-G.; Liu, T.; Lovell, T.; Noodleman, L., DFT Calculations of 57Fe Mössbauer Isomer Shifts and Quadrupole Splittings for Iron Complexes in Polar Dielectric Media: Applications to Methane Monooxygenase and Ribonucleotide Reductase. J. Comput.Chem. 2006, 27, 1292-1306.

38. Hopmann, K. H.; Ghosh, A.; Noodleman, L., DFT Calculations on Mössbauer Parameters of Nonheme Iron Nitrosyls. Inorg. Chem. 2009, 48, 9155-9165.

39. Petrilli, H. M.; Blöchl, P. E.; Blaha, P.; Schwarz, K., Electric-Field-Gradient Calculations Using the Projector Augmented Wave Method. Phys. Rev. B 1998, 57, 1469014697.

40. Peter, B., Calculations of Mössbauer Parameters in Solids by DFT Band Structure Calculations. . J. Phys.: Conf. Ser. 2010, 217, 012009.

41. Casassa, S.; Ferrari, A. M., Calibration of 57Fe Mössbauer Constants by First Principles. Phys. Chem. Chem. Phys. 2016, 18, 10201-10206.

42. Matanovic, I.; Artyushkova, K.; Atanassov, P., Understanding PGM-free Catalysts by Linking Density Functional Theory Calculations and Structural Analysis: Perspectives and Challenges. Curr. Opin. Electrochem. 2018, 9, 137-144.

43. Geudtner, G. e. a., DeMon2k WIREs Computational Molecular Science 2012, 2, 548555. 
44. Kresse, G.; Hafner, J., Ab Initio Molecular Dynamics for Liquid Metals. Phys. Rev. B 1993, 47, 558-561.

45. Kresse, G.; Hafner, J., Ab Initio Molecular-Dynamics Simulation of the Liquid-MetalAmorphous-Semiconductor Transition in Germanium. Phys. Rev. B 1994, 49, 14251-14269.

46. Kresse, G.; Furthmüller, J., Efficiency of Ab-initio Total Energy Calculations for Metals and Semiconductors Using a Plane-wave Basis Set. Comput. Mat. Sci. 1996, 6, 15-50.

47. Kresse, G.; Furthmüller, J., Efficient Iterative Schemes for Ab Initio Total-energy Calculations Using a Plane-wave Basis Set. Phys. Rev. B 1996, 54, 11169-11186.

48. Godbout, N.; Salahub, D. R.; Andzelm, J.; Wimmer, E., Optimization of Gaussiantype Basis Sets for Local Spin Density Functional Calculations. Part I. Boron Through Neon, Optimization Technique and Validation. Can. J. Chem. 1992, 70, 560-571.

49. Wachters, A. J. H., Gaussian Basis Set for Molecular Wavefunctions Containing Third - Row Atoms. J. Chem. Phys. 1970, 52, 1033.

50. Perdew, J. P.; Burke, K.; Ernzerhof, M., Generalized Gradient Approximation Made Simple. Phys. Rev. Lett. 1996, 77, 3865-3868.

51. Zhang, Y.; Yang, W., Comment on "Generalized Gradient Approximation Made Simple". Phys. Rev. Lett. 1998, 80, 890-890.

52. Lee, C.; Yang, W.; Parr, R. G., Development of the Colle-Salvetti Correlation-Energy Formula into a Functional of the Electron Density. Phys. Rev. B 1988, 37, 785-789.

53. Handy, N. C.; Cohen, A. J., Left-right Correlation Energy. Mol. Phys. 2001, 99, 403412.

54. Köster, A. M.; Reveles, J. U.; Del Campo, J. M., Calculation of Exchange-Correlation Potentials with Auxiliary Function Densities. J. Chem. Phys. 2004, 121, 3417-3424.

55. Wu, Q.; Yang, W. J., Empirical Correction to Density Functional Theory for van Der Waals Interactions. . J. Chem. Phys. 2002, 116, 515-524.

56. Krack, M.; Köster, A. M., An Adaptive Numerical Integrator for Molecular Integrals. J. Chem. Phys. 1998, 108, 3226-3234.

57. Blöchl, P. E., Projector Augmented-Wave Method. Phys. Rev. Lett. 1994, B 50, 17953-17979.

58. Kresse, G.; Joubert, J., From Ultrasoft Pseudopotentials to the Projector AugmentedWave Method. Phys. Rev. B 1999, 59, 1758-1775.

59. Varret, F., Mössbauer Spectra of Paramagnetic Powders Under Applied Field: Fe2+ in Fluosilicates. J. Phys. Chem. Solids 1976, 37, 265-271.

60. Champion, P. M.; Chiang, R.; Muenck, E.; Debrunner, P.; Hager, L. P., Moessbauer Investigations of High-spin Ferrous Heme Proteins. II. Chloroperoxidase, Horseradish Peroxidase, and Hemoglobin. Biochemistry 1975, 14, 4159-4166.

61. Charreteur, F.; Jaouen, F.; Ruggeri, S.; Dodelet, J.-P., Fe/N/C Non-precious Catalysts for PEM Fuel Cells: Influence of the Structural Parameters of Pristine Commercial Carbon Blacks on Their Activity for Oxygen Reduction. Electrochim. Acta 2008, 53, 2925-2938.

62. Chung, H. T.; Cullen, D. A.; Higgins, D.; Sneed, B. T.; Holby, E. F.; More, K. L.; Zelenay, P., Direct Atomic-level Insight Into the Active Sites of a High-performance PGMfree ORR Catalyst. Science 2017, 357, 479-484. 
63. Palmer, G., Physical Methods in Bioinorganic Chemistry. University Science Books: Sausalito, CA, USA 2000; p 121-185.

64. Collins, R. L.; Travis, J. C. In The Electric Field Gradient Tensor, Mössbauer Effect Methodology, Boston, MA, Gruverman, I. J., Ed. Springer US: Boston, MA, 1967; pp 123161.

65. Kneebone, J. L.; Daifuku, S. L.; Kehl, J. A.; Wu, G.; Chung, H. T.; Hu, M. Y.; Alp, E. E.; More, K. L.; Zelenay, P.; Holby, E. F.; Neidig, M. L., A Combined Probe-Molecule, Mössbauer, Nuclear Resonance Vibrational Spectroscopy, and Density Functional Theory Approach for Evaluation of Potential Iron Active Sites in an Oxygen Reduction Reaction Catalyst. J. Phys. Chem. C 2017, 121, 16283-16290.

66. Morozan, A.; Sougrati, M. T.; Goellner, V.; Jones, D.; Stievano, L.; Jaouen, F., Effect of Furfuryl Alcohol on Metal Organic Framework-based Fe/N/C Electrocatalysts for Polymer Electrolyte Membrane Fuel Cells. Electrochim. Acta 2014, 119, 192-205.

67. Scherson, D. A.; Fierro, C. A.; Tryk, D.; Gupta, S. L.; Yeager, E. B.; Eldridge, J.; Hoffman, R. W., In-situ Mössbauer Spectroscopy and Electrochemical Studies of the Thermal Stability of Iron Phthalocyanine Dispersed in High Surface Area Carbon. J Electroanal Chem Interfacial Electrochem 1985, 184, 419-426.

68. Wagner, S.; Auerbach, H.; Tait, C. E.; Martinaiou, I.; Kumar, S. C. N.; Kübel, C.; Sergeev, I.; Wille, H.-C.; Behrends, J.; Wolny, J. A.; Schünemann, V.; Kramm, U. I., Elucidating the Structural Composition of an Fe-N-C Catalyst by Nuclear- and ElectronResonance Techniques. Angew. Chem. Int. Ed. 2019, 58, 10486-10492.

69. Yang, H.-J.; Dong, J.; Hong, Y.-H.; Lin, W.-F.; Zhou, Z.-Y.; Sun, S.-G., Comparative Investigation of $\mathrm{CO} 2$ and Oxygen Reduction on Fe/N/C Catalysts. Electrochem. Commun. 2018, 97, 82-86.

70. Malko, D.; Kucernak, A.; Lopes, T., Performance of Fe-N/C Oxygen Reduction Electrocatalysts toward $\mathrm{NO} 2-, \mathrm{NO}$, and $\mathrm{NH} 2 \mathrm{OH}$ Electroreduction: From Fundamental Insights into the Active Center to a New Method for Environmental Nitrite Destruction. $J$. Am. Chem. Soc. 2016, 138, 16056-16068.

71. Li, A.; Nicolae, S. A.; Qiao, M.; Preuss, K.; Szilágyi, P. A.; Moores, A.; Titirici, M.M., Homogenous Meets Heterogenous and Electro-Catalysis: Iron-Nitrogen Molecular Complexes within Carbon Materials for Catalytic Applications. ChemCatChem 2019, 11, 3602-3625.

72. Xie, J.; Yin, K.; Serov, A.; Artyushkova, K.; Pham, H. N.; Sang, X.; Unocic, R. R.; Atanassov, P.; Datye, A. K.; Davis, R. J., Selective Aerobic Oxidation of Alcohols over Atomically-Dispersed Non-Precious Metal Catalysts. ChemSusChem 2017, 10, 359-362.

73. Cui, X.; Li, H.; Wang, Y.; Hu, Y.; Hua, L.; Li, H.; Han, X.; Liu, Q.; Yang, F.; He, L.; Chen, X.; Li, Q.; Xiao, J.; Deng, D.; Bao, X., Room-Temperature Methane Conversion by Graphene-Confined Single Iron Atoms. Chem 2018, 4, 1902-1910.

74. Deng, D; Chen, X.; Yu L.; Wu, X.; Liu, Q.; Yang, H.; Tian, H. Hu, Y.; Du, P.; Si, R.; Wang, J.; Cui, X.; Li, H.; Xiao, J.; Xu, T.; Deng, J.; Yang, F.; Duchesne, P.N.; Zhang, P.; Zhou, J.; Sun, L.; Li, J.; Pan, X.; Bao, X., A Single Iron Site Confined in a Graphene Matrx for the Catalytic Oxidation of Benzene at Room Temperature, Sci. Adv. 2015, 1, 1500462. 